University of Nebraska - Lincoln

DigitalCommons@University of Nebraska - Lincoln

Faculty Publications, Department of Physics and Astronomy

Research Papers in Physics and Astronomy

3-24-2004

\title{
Perturbative analysis of the triply differential cross section and circular dichroism in photo-double-ionization of $\mathrm{He}$
}

Andrei Y. Istomin

University of Nebraska-Lincoln, aistomin2@unl.edu

N. L. Manakov

Voronezh State University, manakov@phys.vsu.ru

Anthony F. Starace

University of Nebraska-Lincoln, astarace1@unl.edu

Follow this and additional works at: https://digitalcommons.unl.edu/physicsfacpub

Part of the Physics Commons

Istomin, Andrei Y.; Manakov, N. L.; and Starace, Anthony F., "Perturbative analysis of the triply differential cross section and circular dichroism in photo-double-ionization of $\mathrm{He}$ " (2004). Faculty Publications, Department of Physics and Astronomy. 19.

https://digitalcommons.unl.edu/physicsfacpub/19

This Article is brought to you for free and open access by the Research Papers in Physics and Astronomy at DigitalCommons@University of Nebraska - Lincoln. It has been accepted for inclusion in Faculty Publications, Department of Physics and Astronomy by an authorized administrator of DigitalCommons@University of Nebraska Lincoln. 


\title{
Perturbative analysis of the triply differential cross section and circular dichroism in photo-double-ionization of $\mathrm{He}$
}

\author{
Andrei Y. Istomin, ${ }^{1}$ N. L. Manakov, ${ }^{2}$ and Anthony F. Starace ${ }^{1}$ \\ ${ }^{1}$ Department of Physics and Astronomy, The University of Nebraska, Lincoln, Nebraska 68588-0111, USA \\ ${ }^{2}$ Physics Department, Voronezh State University, Voronezh 394006, Russia
}

(Received 21 August 2003; published 24 March 2004)

\begin{abstract}
We extend application of our lowest-order perturbative approach (in electron-electron correlation) for analysis of photo-double-ionization (PDI) of He [A.Y. Istomin et al., J. Phys. B 35, L543 (2002)] to excess energies up to $450 \mathrm{eV}$ and to analysis of circular dichroism. We find that account of electron correlation in the final state to first order provides predictions for the triply differential cross section and circular dichroism that are in reasonable agreement with absolute data for excess energies up to $80 \mathrm{eV}$. For an excess energy of $450 \mathrm{eV}$, account of electron correlation in both initial and final states is necessary and the predicted triply differential cross sections are in agreement with absolute data only for large mutual ejection angles. We find that at excess energies of a few tens of eV, the PDI is dominated by the "virtual" knock-out mechanism, while the "direct" (on-shell) knock-out process gives only small contributions for large mutual ejection angles. As a result, we conclude that the circular dichroism effect at these energies originates from the nonzero electron Coulomb phase shifts.
\end{abstract}

DOI: 10.1103/PhysRevA.69.032713

PACS number(s): $32.80 . \mathrm{Fb}$

\section{INTRODUCTION}

Over many decades both the theoretical analysis and the experimental measurement of single-photon, doubleionization (PDI) processes, especially for the He atom, have been of intense interest [1-3]. Owing to the difficulty of describing the six-dimensional double-continuum final state, most theoretical treatments have employed significant approximations. Initially, theorists employed ground-state wave functions and uncorrelated final-state wave functions calculated in the field of the doubly charged $(Z=2)$ He nucleus $[4,5]$. By the mid-1970s, however, theorists shifted to perturbation theory treatments (using either Coulomb [6-8] or, more recently, various forms of Hartree-Fock [9-12] basis functions) in order to introduce correlation effects between the two ionized electrons. All of these theoretical treatments have focused on the total cross section for double ionization of He. It is only in the past decade or so that attention has shifted primarily to the triply differential cross section (TDCS), which describes the angular distribution of the two ionized electrons and which is a much more sensitive test of theoretical approximations and models. These more recent theoretical treatments of the differential and total cross sections have reverted to using correlated or uncorrelated ground-state wave functions and different kinds of improved analytical final-state wave functions, including the so-called 3C (three Coulomb) functions [13] (which satisfy the proper asymptotic boundary conditions for double ionization [14-21]), independent-particle final-state wave functions calculated in the field of momentum-dependent effective Coulomb charges [22,23], and modified 3C functions that involve momentum-dependent effective charges [21,24]. In general, even though the TDCS angular patterns are reproduced qualitatively, in those works where comparison with absolute experimental data is made (see, e.g., Refs. [20,24]), various scaling factors had to be introduced; also, the results of these treatments depend significantly upon the gauge employed. Perturbative treatment of the TDCS was discussed in general in Ref. [25], but an actual calculation using lowestorder perturbation theory (LOPT) with a basis of $Z=2$ Coulomb functions was only carried out recently [26]. The recent availability of absolute experimental data for the TDCS [20,27-31], although for a limited number of photon energies, has stimulated also nonperturbative numerical theoretical treatments of the two-electron correlations. The eigenchannel $R$-matrix method [32], the hyperspherical closecoupling method [33], and the convergent close-coupling (CCC) method [34] were used to evaluate the total doubleionization cross section and the ratio of double- to singleionization cross sections. The TDCSs have been calculated using the CCC method [29,35,36], the hyperspherical $R$-matrix method with semiclassical outgoing waves (HRMSOW) [37], and the time-dependent close-coupling (TDCC) method [38], which involves direct solution of the timedependent Schrödinger equation.

Much has been learned from these nearly four decades of theoretical analyses; we note here several aspects which motivate the present work. First, the double-ionization process is highly sensitive to the gauge in which the electric dipole interaction is evaluated [11,16,21,39]; for low photon energies the velocity gauge is least sensitive to higher-order perturbative corrections [9] and is found to give the best absolute values for the TDCS [21]. Second, the TDCS is very sensitive to final-state (FS) correlations (in all gauges), and for the case of equal energy sharing is not sensitive to the precise form of the initial-state wave function [21]. [Indeed, the total double-ionization cross section for low photon energies $(\leqslant 500 \mathrm{eV})$ may be described quite accurately (in the velocity gauge) by taking only final-state correlations into account [11].] Third, the detailed perturbation theory analyses of Refs. [9] (for $\omega \leqslant 290 \mathrm{eV}$ ) and [12] (for $\omega \leqslant 14 \mathrm{keV}$ ) provide much information on approximate ways to take into 
account high-order correlation terms. Specifically, many higher-order FS correlation terms that have to do with electron screening effects can be taken into account by using two different basis sets for the ionized electrons, with the faster electron seeing a net charge of $Z=1$ and the slower electron seeing a net charge of $Z=2$. (Reference [9] suggests that if a single basis set is to be employed, it should probably have $1 \leqslant Z \leqslant 2$; however, no calculations for such a single set were carried out.) Regarding ground-state (GS) correlations, Ref. [9] indicates that higher-order terms tend to cancel the lowest-order GS correlation diagram, particularly when a basis of $Z=2$ wave functions is employed.

Recently, Keller [26] has reported the first LOPT calculation of the TDCS for double photoionization of He using a basis of $Z=2$ Coulomb functions. For the case of symmetric energy sharing there is qualitative agreement with experiment, while for asymmetric energy sharing there are qualitative discrepancies with experiment. In all cases, the theoretical results must be scaled by factors ranging from 0.10 to 0.19 in order to be compared with the absolute experimental data [29]. Because for the single-particle binding energies in intermediate states a value equal to one half (i.e., $39.5 \mathrm{eV}$ ) the experimental double-ionization energy $(79 \mathrm{eV})$ was used [26] (instead of using the theoretical Coulomb binding energy $-Z^{2} / 2$ ), the results of these calculations should be gauge dependent even if all LOPT diagrams are included. Within the velocity gauge, which is the only one employed, the GS correlations are predicted to have only a small influence on the TDCS relative to FS correlations.

We have recently presented results of another set of calculations of the TDCSs using a LOPT account of electronelectron interactions [40]. As shown in Ref. [40], account of final-state correlations to lowest order, combined with an account of electron screening in the ground state, provides TDCSs that are in excellent agreement with both absolute experimental data and with accurate theoretical results for an excess energy of $20 \mathrm{eV}$. The range of the excess energies over which the approach in Ref. [40] is applicable remains an open question.

In the present work we provide a detailed presentation of the theoretical approach for the double ionization TDCS for He that was only sketched briefly in Ref. [40]. We also analyze the predictions of this approach over a broad energy range (up to excess energies of $450 \mathrm{eV}$ ). Finally, we analyze in detail the circular dichroism (CD) effect in the TDCS for double ionization of He, i.e., the fact that the TDCS is different for right- and left-circularly polarized light. We find that LOPT account of final-state electron correlation, combined with variational account of electron screening in the ground state, provides predictions for the TDCS that agree reasonably well with both experimental data and the most accurate $a b$ initio theoretical results for excess energies up to $80 \mathrm{eV}$. At higher excess energies, such as at $450 \mathrm{eV}$, account of both ground-state and final-state correlations is necessary; we find that LOPT provides predictions for the TDCS that are in reasonable agreement with experimental data and accurate theoretical results only for large mutual ejection angles. Analysis of our CD results shows that (for excess energies of the order of tens of $\mathrm{eV}$ ) the $\mathrm{CD}$ effect originates from nonzero Coulomb phase shifts. Our predictions for CD are in excellent agreement with absolute experimental data for an excess energy of $20 \mathrm{eV}$.

\section{THEORY}

\section{A. General results}

Using the velocity gauge for the electric dipole interaction of atomic electrons with a photon having the frequency $\omega$ and the unit (in general, complex) polarization vector e $\left(\mathbf{e} \cdot \mathbf{e}^{*}=1\right)$, the TDCS for PDI from $S$ states of He or He-like ions has the following form:

$$
\sigma_{T D C S} \equiv \frac{d^{3} \sigma}{d \Omega_{1} d \Omega_{2} d E_{1}}=C_{0}\left|M\left(\mathbf{p}_{1}, \mathbf{p}_{2}, \mathbf{e}\right)\right|^{2},
$$

where $\mathbf{p}_{1}$ and $\mathbf{p}_{2}$ are the photoelectron momenta, $C_{0}$ $=\left(4 \pi^{2} \alpha p_{1} p_{2}\right) / \omega$ is an overall constant factor (where $\alpha$ $=1 / 137)$, and $M\left(\mathbf{p}_{1}, \mathbf{p}_{2}, \mathbf{e}\right)$ is the transition amplitude,

$$
M\left(\mathbf{p}_{1}, \mathbf{p}_{2}, \mathbf{e}\right)=\left\langle\psi_{\mathbf{p}_{1} \mathbf{p}_{2}}\left|\mathbf{e} \cdot\left(-i \boldsymbol{\nabla}_{1}-i \nabla_{2}\right)\right| \psi_{E_{0}}\right\rangle .
$$

In Eq. (2), $\psi_{E_{0}}\left(\mathbf{r}_{1}, \mathbf{r}_{2}\right)$ is the ground-state wave function and $\psi_{\mathbf{p}_{1} \mathbf{p}_{2}}\left(\mathbf{r}_{1}, \mathbf{r}_{2}\right)$ is the wave function for the final two-electron continuum state, which we assume to be normalized according to $\left\langle\psi_{\mathbf{p}_{1} \mathbf{p}_{2}} \mid \psi_{\mathbf{p}_{1}^{\prime} \mathbf{p}_{2}^{\prime}}\right\rangle=\delta\left(\mathbf{p}_{1}-\mathbf{p}_{1}^{\prime}\right) \delta\left(\mathbf{p}_{2}-\mathbf{p}_{2}^{\prime}\right)$. (Atomic units are used through this paper, unless otherwise stated.) Since we consider PDI from the ${ }^{1} S$ state and since the photoion has zero angular momentum, the PDI amplitude is a rotationally invariant scalar and its general form follows from general symmetry arguments [23],

$$
M=\left(\mathbf{e} \cdot \hat{\mathbf{p}}_{1}\right) f\left(p_{1}, p_{2}, \cos \theta\right)+\left(\mathbf{e} \cdot \hat{\mathbf{p}}_{2}\right) f\left(p_{2}, p_{1}, \cos \theta\right) .
$$

Thus, the entire dynamics of the PDI process for a He-like system is completely described by a single scalar function, the polarization-independent amplitude $f$, which depends on the absolute values of the two photoelectron momenta and their mutual angle $\theta_{12} \equiv \theta$, where $\cos \theta=\hat{\mathbf{p}}_{1} \cdot \hat{\mathbf{p}}_{2}$. [Note that for a fixed excess energy $E$ (where $E=E_{0}+\omega=p_{1}^{2} / 2+p_{2}^{2} / 2$ ), $f$ depends only on two independent variables.] The general form of the angular dependence of the invariant amplitude $f$ is given by its multipole expansion in terms of derivatives of the Legendre polynomials, $P_{l}^{\prime}(\cos \theta)$ $=d P_{l}(\cos \theta) / d \cos \theta[41]$,

$$
\begin{aligned}
f\left(p, p^{\prime}, \cos \theta\right)= & \sum_{l_{1}=0}^{\infty}(-1)^{l_{1}}\left(\sum_{l_{2}=l_{1} \pm 1} \frac{\left\langle p, p^{\prime} ;\left(l_{1}, l_{2}\right) 1\|\mathbf{D}\| 0\right\rangle}{\sqrt{\max \left(l_{1}, l_{2}\right)}}\right) \\
& \times P_{l_{1}}^{\prime}(\cos \theta),
\end{aligned}
$$

where $\left\langle p, p^{\prime} ;\left(l_{1}, l_{2}\right) 1|| \mathbf{D}|| 0\right\rangle$ is a two-electron reduced matrix element of the dipole operator between the ${ }^{1} S$ ground state and the ${ }^{1} P$ final (two-electron) continuum state, $\left(p, p^{\prime}\right)$ $=\left(p_{1}, p_{2}\right)$ or $\left(p_{2}, p_{1}\right)$, and $l_{1}$ and $l_{2}$ are the individual orbital angular momenta of the two photoelectrons, which couple to the total orbital angular momentum $L=1$. Substituting Eq. (3) into Eq. (1) we obtain an expression for the TDCS for the general case of an elliptically polarized photon as the sum of four polarization- and angular-dependent terms [41], 


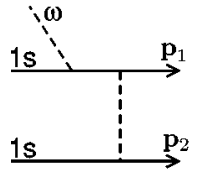

(a) FSC

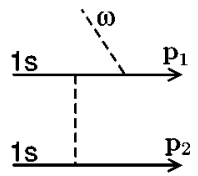

(b) GSC
FIG. 1. Schematic diagrams contributing to the PDI process in the first order of perturbation theory. (a) FSC, final-state correlations and (b) GSC, ground-state correlations. Two additional diagrams with exchanged $\mathbf{p}_{1}$ and $\mathbf{p}_{2}$ must be included in the transition amplitude.

$$
\begin{aligned}
\sigma_{T D C S}= & C_{0}\left\{\left|f_{1}\right|^{2}\left|\mathbf{e} \cdot \hat{\mathbf{p}}_{1}\right|^{2}+\left|f_{2}\right|^{2}\left|\mathbf{e} \cdot \hat{\mathbf{p}}_{2}\right|^{2}\right. \\
& +2 \operatorname{Re}\left\{f_{1} f_{2}^{*}\right\} \operatorname{Re}\left\{\left(\mathbf{e} \cdot \hat{\mathbf{p}}_{1}\right)\left(\mathbf{e}^{*} \cdot \hat{\mathbf{p}}_{2}\right)\right\} \\
& \left.+\xi \operatorname{Im}\left\{f_{1} f_{2}^{*}\right\} \hat{\mathbf{k}} \cdot\left[\hat{\mathbf{p}}_{1} \times \hat{\mathbf{p}}_{2}\right]\right\},
\end{aligned}
$$

where $f_{1} \equiv f\left(p_{1}, p_{2}, \cos \theta\right), f_{2} \equiv f\left(p_{2}, p_{1}, \cos \theta\right), \hat{\mathbf{k}}$ is the unit vector in the direction of the photon beam, and $\xi$ $=i \hat{\mathbf{k}} \cdot\left[\mathbf{e} \times \mathbf{e}^{*}\right]$ is the degree of circular polarization of the photon. The last term in Eq. (5) describes the dependence of the TDCS upon the photon helicity, i.e., the CD effect $[15,41]$. The parametrization of the transition amplitude and the TDCS in terms of the single function $f\left(p, p^{\prime}, \cos \theta\right)$ [which is different for the sets $\left(p, p^{\prime}\right)$ $=\left(p_{1}, p_{2}\right)$ and $\left.\left(p_{2}, p_{1}\right)\right]$ is equivalent to that in terms of the symmetrized amplitudes, $a_{\mathrm{g}}$ and $a_{\mathrm{u}}[42,43]$, which are, respectively, symmetric and antisymmetric in the interchange of electron momenta, and which may be expressed in terms of the amplitude $f$ as follows:

$$
a_{\mathrm{g}, \mathrm{u}}=\left[f\left(p_{1}, p_{2}, \cos \theta\right) \pm f\left(p_{2}, p_{1}, \cos \theta\right)\right] / 2 \equiv\left(f_{1} \pm f_{2}\right) / 2 .
$$

Though this parametrization does not simplify the general analysis, it is particularly convenient for the case of equal energy sharing, $p_{1}=p_{2}$, when $f_{1}=f_{2}$ and $a_{u}$ vanishes. Multipole expansions of the symmetrized amplitudes equivalent to Eq. (4) were obtained in Ref. [43].

\section{B. LOPT approach for account of electron correlations}

Equations (3)-(6) are very general and independent of the dynamical model used to describe correlated electron motion in both the ground state and the two-electron continuum [or to estimate the two-electron dipole matrix elements in Eq. (4)]. In this work, we employ the simplest approximation to evaluate the polarization-invariant amplitude $f\left(p, p^{\prime}, \cos \theta\right)$, that is, we use the LOPT in the interelectron interaction $\left(1 / r_{12}\right)$ to account for electron correlations in the ground state $\psi_{E_{0}}\left(\mathbf{r}_{1}, \mathbf{r}_{2}\right)$ and in the two-electron continuum state $\psi_{\mathbf{p}_{1} \mathbf{p}_{2}}\left(\mathbf{r}_{1}, \mathbf{r}_{2}\right)$ in the transition amplitude (2). In LOPT using a basis of one-electron Coulomb orbitals for a nuclear charge $Z$, the total amplitude (2) for a $\mathrm{He}$ or a He-like ion can be schematically represented by four diagrams, in which the electron correlation interaction is taken into account either in the final state [Fig. 1(a)] or in the ground state [Fig. 1(b)]. The direct amplitudes are depicted in Fig. 1; the exchange amplitudes have final electron momenta $\mathbf{p}_{1}$ and $\mathbf{p}_{2}$ interchanged. Analytical expressions for the amplitudes presented in Fig. 1 are

$$
\begin{gathered}
M_{F}=-i \sqrt{2}\left\langle\psi_{\mathbf{p}_{1}}^{(-)}\left|U_{\mathbf{p}_{2}}^{(-)}(\mathbf{r}) G_{E_{F}}\left(\mathbf{r}, \mathbf{r}^{\prime}\right)\left(\mathbf{e} \cdot \nabla_{\mathbf{r}^{\prime}}\right)\right| \varphi_{0}\right\rangle, \\
M_{G}=-i \sqrt{2}\left\langle\psi_{\mathbf{p}_{1}}^{(-)}\left|\left(\mathbf{e} \cdot \nabla_{\mathbf{r}}\right) G_{E_{G}}\left(\mathbf{r}, \mathbf{r}^{\prime}\right) U_{\mathbf{p}_{2}}^{(-)}\left(\mathbf{r}^{\prime}\right)\right| \varphi_{0}\right\rangle, \\
U_{\mathbf{p}_{2}}^{(-)}(\mathbf{r})=\left\langle\psi_{\mathbf{p}_{2}}^{(-)}\left|\frac{1}{\left|\mathbf{r}-\mathbf{r}^{\prime}\right|}\right| \varphi_{0}\right\rangle,
\end{gathered}
$$

where $G_{E_{F}}$ and $G_{E_{G}}$ are one-particle Coulomb Green Functions (CGFs) with energy parameters $E_{F}=E_{p_{1}}+E_{p_{2}}-\epsilon_{1 s}$ and $E_{G}=2 \epsilon_{1 s}-E_{p_{2}}\left(\right.$ where $\left.E_{p_{i}} \equiv p_{i}^{2} / 2\right)$; the functions $\varphi_{0}$ and $\psi_{\mathbf{p}}^{(-)}$ are one-particle Coulomb wave functions of a hydrogenlike ion ground and continuum states [the function $\psi_{\mathbf{p}}^{(-)}$is normalized according to $\left.\left\langle\psi_{\mathbf{p}} \mid \psi_{\mathbf{p}^{\prime}}\right\rangle=\delta\left(\mathbf{p}-\mathbf{p}^{\prime}\right)\right]$; and the factor $\sqrt{2}$ in Eqs. (7) and (8) accounts for symmetrization of the final state. The two amplitudes that correspond to the exchange diagrams are obtained by interchanging momentum vectors $\mathbf{p}_{1}$ and $\mathbf{p}_{2}$ in Eqs. (7)-(9) and using $E_{G}=2 \epsilon_{1 s}-E_{p_{1}}$.

Use of the LOPT approach to account for electron correlations in PDI of He for an excess energy of the order of tens of $\mathrm{eV}$ reveals a number of difficulties compared to previous applications of LOPT to describe the PDI process at high excess energies (see, e.g., Refs. [7,25,44]). The first difficulty is that if one employs a single-particle Coulomb basis set using the bare charge of the He nucleus, $Z=2$, the theoretical zero-order ground-state energy of $\mathrm{He}\left(\left|E^{\text {th }}\right|=2\left|\epsilon_{1 s}\right|=Z^{2} / 2\right.$ $=108.84 \mathrm{eV})$ is very different from the experimental value $\left(\left|E^{\text {expt }}\right|=79.02 \mathrm{eV}\right)$. One way to bypass this problem is to use a Coulomb basis set with an effective, e.g., variational, screened charge $Z_{s}$. (This approach was used in Ref. [40].) Another ( $a d h o c$ ) way would be to set the one-electron ground-state binding energy equal to one half the experimental two-electron binding energy, $\left|E^{\text {expt }}\right|$, i.e., $\epsilon_{1 s}=E^{\text {expt }} / 2$, and to use these values in the energy parameters $E_{F}$ and $E_{G}$ of the CGFs in Eqs. (7) and (8). [This approach, i.e., using $\epsilon_{1 s} \equiv$ $-(79.02 / 2) \mathrm{eV}$, was used in Ref. [26].] However, if one is mostly concerned about PDI of He by high-energy photons (as well as of He-like ions with higher nuclear charge, $Z$ $\geqslant 2$ ), the discrepancy between the theoretical and experimental energies becomes far less significant. A second difficulty in direct application of a LOPT approach to the analysis of the PDI process lies in the fact that for excess energies of the order of tens of $\mathrm{eV}$, the LOPT GSC amplitude, which is evaluated using an uncorrelated final state (and whose predicted angular distribution is very different from experiment), overestimates the role of GSCs, i.e., its effect is reduced when higher-order GSC terms are taken into account. In order to bypass these two difficulties, we use two substantially different models for taking electron correlation into account for the cases of intermediate (i.e., tens of $\mathrm{eV}$ ) and high (hundreds of eV) excess energies. 

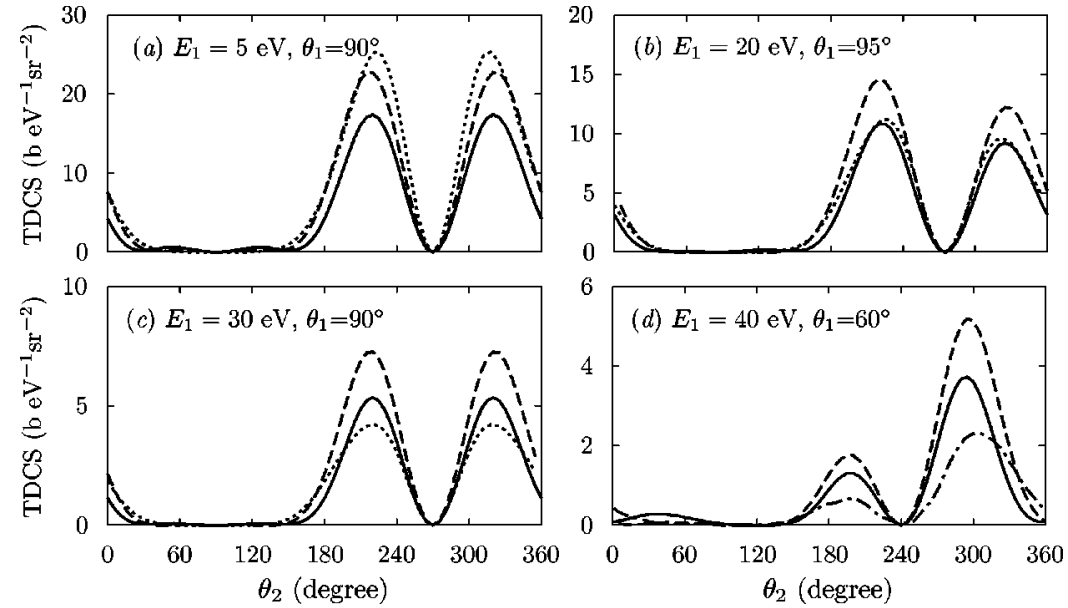

FIG. 2. TDCS for double photoionization of $\mathrm{He}$ at an excess energy of (a) $25 \mathrm{eV}$, (b) $40 \mathrm{eV}$, (c) $60 \mathrm{eV}$, and (d) $80 \mathrm{eV}$. Full curves, present LOPT results with account of FSC and all individual orbital angular momenta of the two photoelectrons, for $Z_{s}=27 / 16$ in the ground state and $Z=2$ in intermediate and final states; dashed curves, same as above but for a single basis set, with $Z_{s}=27 / 16$ in all states; dotted curves, results of the TDCC calculations of Colgan and Pindzola [45]; dot-dashed curves, results of the CCC calculations in Ref. [46].

\section{Model for intermediate excess energies}

In the case of intermediate excess energies, we account for electron screening in the ground state by using the wellknown variationally determined effective charge, $Z_{s}=Z$ $-5 / 16$, and take final-state electron correlation into account to first order. (For He, use of $Z_{s}=27 / 16$ gives $\left|E^{t h}\right|$ $\approx 77.5 \mathrm{eV}$, which is close to the experimental value of $79.02 \mathrm{eV}$.) The question now arises as to what basis set should one use to represent intermediate and final states in this model. In any perturbation theory approach it is usually preferable to employ a single one-electron basis set of states, in order to avoid having to deal with nonorthogonality between orbitals belonging to different basis sets as well as to have gauge-invariant transition amplitudes. Therefore, if one uses an effective charge of $Z_{s}=27 / 16$ in defining the groundstate one-electron functions, in order to maintain both orthogonality of the one-electron orbitals and gauge invariance, one should use this value to describe all excited and continuum one-electron basis functions. Physically, however, as the photon energy increases, the escaping electrons spend less time near the nucleus and thus their mutual screening becomes less significant; asymptotically, of course, each electron sees an effective nuclear charge of 2. Thus, on physical grounds it would seem that as the photon energy increases, the use of two basis sets becomes physically more appropriate: for the ground state, a set of one-electron orbitals calculated for an effective nuclear charge $Z_{s}=27 / 16$ and for intermediate and final states, a set of one-electron orbitals calculated for the bare nuclear charge, $Z=2$. Of course, any complete basis set will do if one treats electron correlations to high order; but if, as here, one wishes to treat final-state correlations in lowest order, then the choice of basis set becomes very important. Also, we employ the velocity gauge, for the reasons discussed in the introduction above (i.e., it is the least sensitive and provides the best absolute values). As has been shown in Ref. [40], the first approach (which uses a single basis set) provides TDCSs that are in excellent agreement with available absolute experimental data for an excess energy of $20 \mathrm{eV}$. However, we have found that the second approach, which uses the $Z_{s}=27 / 16$ basis set in the ground state only and which is somewhat more consistent from a physical point of view, provides better agreement for higher excess energies, up to $80 \mathrm{eV}$. (The TDCSs obtained using these two approaches are compared with results of accurate $a b$ initio calculations of Colgan and Pindzola [45] and Kheifets and Bray [46] in Fig. 2.) Therefore, for excess energies in the range of $20 \mathrm{eV}$ to $80 \mathrm{eV}$ we employ the approach which uses the $Z_{s}=27 / 16$ basis set for ground-state orbitals, and the $Z=2$ basis set for intermediate and final-state orbitals of $\mathrm{He}$. Our approach corresponds to an assumption (which is supported by good agreement with experimental data and with other $a b$ initio calculations) that, for the excess energies considered, the PDI process is dominated (in the velocity gauge) by final-state correlations (i.e., the TS-1 mechanism dominates) and that the entire effect of electron-nucleus and electron-electron interactions in the ground state may be taken into account on the level of screening effects, by using the "screening charge," $Z_{s}=Z-5 / 16$. Thus, for intermediate excess energies, we approximate the total amplitude (2) by the sum of two matrix elements, $M_{F}\left(p_{1}, p_{2}\right)$ and $M_{F}\left(p_{2}, p_{1}\right)$, and use in Eqs. (7) and (9) the following form for the variational ground-state orbitals $\varphi_{0}(r)$,

$$
\varphi_{0}(r)=\sqrt{Z_{s}^{3} / \pi} \exp \left(-Z_{s} r\right)
$$

\section{Model for high excess energies}

In the case of high excess energies (of the order of a few hundred $\mathrm{eV}$ ), the contributions of electron correlations in initial and final states are equally important. Therefore at high photon energies we take into account both FS and GS correlation amplitudes, $M_{F}$ and $M_{G}$, and use the bare nuclear charge $Z$ in all basis states. This approach provides gaugeindependent predictions for the TDCS. If the theoretical value for the one-particle ground-state energy $\epsilon_{1 s}=-Z^{2} / 2$ is used (as in our approach), the transition amplitude, $M=M_{F}$ 
$+M_{G}$, is gauge invariant, while the separate amplitudes $M_{F}$ and $M_{G}$ are gauge dependent. It is easy to show that the amplitudes $M_{F, G}\left(\mathbf{p}_{1}, \mathbf{p}_{2}\right)$ [or $\left.M_{F, G}\left(\mathbf{p}_{2}, \mathbf{p}_{1}\right)\right]$ in the $L$ and $V$ gauges are related as follows:

$$
\begin{gathered}
M_{F}^{V}=i \omega_{f i} M_{F}^{L}+i \Delta M, \\
M_{G}^{V}=i \omega_{f i} M_{G}^{L}-i \Delta M, \\
\Delta M=\left\langle\psi_{\mathbf{p}_{1}}^{(-)} \psi_{\mathbf{p}_{2}}^{(-)}\left|\mathbf{e} \cdot\left(\mathbf{r}_{1}+\mathbf{r}_{2}\right) / r_{12}\right| \varphi_{0} \varphi_{0}\right\rangle,
\end{gathered}
$$

where $\omega_{f i}=E_{1}+E_{2}-2 \epsilon_{1 s}$. If one uses values other than the theoretical ones for the one-electron binding energy (e.g., $E^{\exp t} / 2$, as done in Ref. [26]), the gauge invariance of $M$ is lost and the following relation holds:

$$
M_{F}^{V}+M_{G}^{V}=i \omega M_{G}^{L}+i\left(\omega+E^{e x p t} / 2-\epsilon_{1 s}\right) M_{F}^{L} .
$$

[Obviously, in our approach for intermediate energies (discussed above) the results are gauge-dependent (because we use two different basis sets and account only for final-state correlations). As noted already, we employ the velocity gauge for intermediate energies, as this one is the least sensitive to higher-order correlation effects and is in better agreement with experiment and ab initio calculations (as discussed in the Introduction and in Ref. [40]).]

\section{Scaling properties}

Although in the present paper we only discuss results for neutral $\mathrm{He}$, we emphasize that LOPT calculations are most appropriate for He-like ions with higher $Z$ because the omitted high-order correlation corrections have a relative magnitude of the order of $Z^{-1}$. Thus the LOPT results are more accurate for high- $Z$ He-like ions (including for the groundstate energy). We note also that the results of LOPT calculations (using a single basis set) can be easily $Z$ scaled for application to PDI of highly charged He-like ions, as follows [47]:

$$
\sigma_{T D C S}\left[E_{1}, E_{2}, Z\right]=\frac{1}{Z^{6}} \sigma_{T D C S}\left[\frac{E_{1}}{Z^{2}}, \frac{E_{2}}{Z^{2}}, 1\right] .
$$

\section{Treatment of photoelectron orbital angular momenta}

Finally, in order to evaluate the matrix elements (7)-(9) we use two different approaches: (i) conventional partialwave expansions for $1 / r_{12}$, the CGF, and the Coulomb continuum states, taking only $s, p, d$, and $f$ electron partial waves into account; (ii) exact account of all individual photoelectron angular momenta by using a closed form for the Coulomb continuum states, a representation for the CGF in parabolic coordinates, and an integral representation for $1 / r_{12}$. In both cases we present general derivations for $M_{F}$ using Eq. (10) to represent the ground-state orbitals, while for $M_{G}$ we use Eq. (10) with $Z_{s}=Z$. Detailed descriptions of each of these two methods for treating photoelectron orbital angular momenta are given in the following two sections.

\section{Partial-wave expansions of the PDI amplitude}

In our first approach we use partial-wave expansions for the continuum Coulomb wave functions, for the CGF, and for $1 / r_{12}$ in Eqs. (7)-(9),

$$
\begin{gathered}
\psi_{\mathbf{p}}^{(-)}(\mathbf{r})=\frac{(2 \pi)^{-3 / 2}}{2 p} \sum_{l=0}^{\infty} i^{l}(2 l+1) e^{-i \delta_{l}(p)} R_{p l}(r) P_{l}(\hat{\mathbf{p}} \cdot \hat{\mathbf{r}}), \\
G_{E}\left(\mathbf{r}, \mathbf{r}^{\prime}\right)=\sum_{l, m} g_{l}\left(E ; r, r^{\prime}\right) Y_{l m}(\hat{\mathbf{r}}) Y_{l m}^{*}\left(\hat{\mathbf{r}}^{\prime}\right), \\
\frac{1}{\left|\mathbf{r}-\mathbf{r}^{\prime}\right|}=\sum_{l=0}^{\infty} \frac{r_{<}^{l}}{r_{>}^{l+1}} P_{l}\left(\hat{\mathbf{r}} \cdot \hat{\mathbf{r}}^{\prime}\right),
\end{gathered}
$$

where $\delta_{l}(p)=\arg \Gamma(l+1+i \eta)$ is a Coulomb phase shift, $\eta$ $=Z / p$, and $r_{<}=\min \left(r, r^{\prime}\right), r_{>}=\max \left(r, r^{\prime}\right) . R_{p l}(r)$ is the radial part of the Coulomb wave function,

$$
R_{p l}(r)=\frac{C_{p l}(2 p r)^{l}}{(2 l+1) !} e^{-i p r} \Phi(i \eta+l+1,2 l+2,2 i p r),
$$

where $\Phi$ is a confluent hypergeometric function and $C_{p l}$ $=2 p \exp (\pi \eta / 2)|\Gamma(l+1+i \eta)|$. The radial function (19) is normalized as follows: $\int_{0}^{\infty} R_{p l}(r) R_{p^{\prime} l}(r) r^{2} d r=2 \pi \delta\left(p-p^{\prime}\right)$. For the radial part of the CGF we use the integral representation [48]

$$
\begin{aligned}
g_{l}\left(E ; r, r^{\prime}\right)= & \frac{2}{\sqrt{r r^{\prime}}} \int_{0}^{1} \frac{d u}{1-u} u^{-Z \nu-1 / 2} \\
& \times \exp \left\{-\frac{r+r^{\prime}}{\nu} \frac{1+u}{1-u}\right\} I_{2 l+1}\left(\frac{4 \sqrt{r r^{\prime} u}}{\nu(1-u)}\right),
\end{aligned}
$$

where $\nu=1 / \sqrt{-2 E}(\nu=i|\nu|$ for $E>0)$ and $I_{n}(x)$ is a modified Bessel function. With the above definitions, the transition amplitude (2) may be expressed as an infinite sum over partial-wave amplitudes,

$$
\begin{aligned}
M= & \sum_{l_{1}, l_{2}=0}^{\infty}\left[M_{F}^{\left(l_{1} l_{2}\right)}\left(p_{1}, p_{2}\right)+M_{G}^{\left(l_{1} l_{2}\right)}\left(p_{1}, p_{2}\right)+M_{F}^{\left(l_{1} l_{2}\right)}\left(p_{2}, p_{1}\right)\right. \\
& \left.+M_{G}^{\left(l_{1} l_{2}\right)}\left(p_{2}, p_{1}\right)\right]
\end{aligned}
$$

where only $l_{2}=l_{1} \pm 1$ contribute, owing to the $P$ symmetry of the final state. Each term $M_{F, G}^{\left(l_{1}, l_{2}\right)}\left(p_{1}, p_{2}\right)$ in Eq. (21) corresponds to the $l_{1}, l_{2}$ component of Eqs. (7) and (8), in which only the angular momenta $l_{1}$ and $l_{2}$ in Eq. (16) for $\psi_{\mathbf{p}_{1}}^{(-)}$and $\psi_{\mathbf{p}_{2}}^{(-)}$, respectively, are retained. The angular integrations in each partial-wave amplitude of Eq. (7), which involve three Legendre polynomials, can be evaluated using the formula 


$$
\begin{aligned}
\int P_{l_{1}}(\hat{\mathbf{a}} \cdot \hat{\mathbf{r}}) P_{l_{2}}(\hat{\mathbf{b}} \cdot \hat{\mathbf{r}})(\hat{\mathbf{c}} \cdot \hat{\mathbf{r}}) d \hat{\mathbf{r}} \\
=\frac{-4 \pi C_{l_{1} 0 l_{2} 0}^{10}}{\sqrt{3 l_{>}\left(2 l_{1}+1\right)\left(2 l_{2}+1\right)}}\left[(-1)^{l_{1}} P_{l_{1}}^{\prime}(\hat{\mathbf{a}} \cdot \hat{\mathbf{b}})(\hat{\mathbf{c}} \cdot \hat{\mathbf{a}})\right. \\
\left.\quad+(-1)^{l_{2}} P_{l_{2}}^{\prime}(\hat{\mathbf{a}} \cdot \hat{\mathbf{b}})(\hat{\mathbf{c}} \cdot \hat{\mathbf{b}})\right],
\end{aligned}
$$

where $l_{2}=l_{1} \pm 1, l_{>}=\max \left(l_{1}, l_{2}\right), \hat{\mathbf{a}}, \hat{\mathbf{b}}$, and $\hat{\mathbf{c}}$ are arbitrary unit vectors, and $P_{l}^{\prime}(x)$ is the derivative of the Legendre polynomial. This formula was obtained by using the reduction formula (C2) for bipolar harmonics of rank 1 given in Ref. [41]. The angular integrations in Eq. (8) which involve the $\nabla$ operator are evaluated using the Wigner-Eckart theorem [49] and the formula (C2) of Ref. [41]. As a result, each term of the series (21) can be written as follows:

$$
M_{F, G}^{\left(l_{1} l_{2}\right)}\left(\mathbf{p}_{1}, \mathbf{p}_{2}\right)=C^{\left(l_{1} l_{2}\right)}\left(\mathbf{e}, \hat{\mathbf{p}}_{1}, \hat{\mathbf{p}}_{2}\right) D_{F, G}^{\left(l_{1} l_{2}\right)}\left(p_{1}, p_{2}\right),
$$

where the angular dependence of the amplitudes is given by

$$
\begin{aligned}
C^{\left(l_{1} l_{2}\right)}\left(\mathbf{e}, \hat{\mathbf{p}}_{1}, \hat{\mathbf{p}}_{2}\right)= & (-1)^{l_{1}} P_{l_{1}}^{\prime}\left(\hat{\mathbf{p}}_{1} \cdot \hat{\mathbf{p}}_{2}\right)\left(\mathbf{e} \cdot \hat{\mathbf{p}}_{1}\right) \\
& +(-1)^{l_{2}} P_{l_{2}}^{\prime}\left(\hat{\mathbf{p}}_{1} \cdot \hat{\mathbf{p}}_{2}\right)\left(\mathbf{e} \cdot \hat{\mathbf{p}}_{2}\right) .
\end{aligned}
$$

The dynamical factors $D_{F, G}^{\left(l_{1} l_{2}\right)}\left(p_{1}, p_{2}\right)$ are proportional to radial matrix elements in which all radial integrals are evaluated analytically using integral representations for the radial parts of the CGF [Eq. (20)] and of one Coulomb wave function $R_{p l}(r)$ (see Appendix A). As a result, the final expressions for $D_{F, G}^{\left(l_{1} l_{2}\right)}\left(p_{1}, p_{2}\right)$, derived in Appendix A, are expressed in terms of two-dimensional integrals that are to be evaluated numerically.

Equations (21), (23), and (24) give the same angular dependence for the amplitude $f$ as the ab initio expression (4). The final expression for the polarization-invariant amplitude $f_{F, G}\left(p_{1}, p_{2}, \cos \theta\right)$ in which either FS or GS correlation is taken into account, is

$$
\begin{aligned}
f_{F, G}\left(p_{1}, p_{2}, \cos \theta\right)= & \sum_{l_{1}, l_{2}=0}^{\infty}\left\{(-1)^{l_{1}} D_{F, G}^{\left(l_{1} l_{2}\right)}\left(p_{1}, p_{2}\right) P_{l_{1}}^{\prime}(\cos \theta)\right. \\
& \left.+(-1)^{l_{2}} D_{F, G}^{\left(l_{1} l_{2}\right)}\left(p_{2}, p_{1}\right) P_{l_{2}}^{\prime}(\cos \theta)\right\} .
\end{aligned}
$$

The amplitude which accounts for both FS and GS correlations is given by

$$
f\left(p_{1}, p_{2}, \cos \theta\right)=f_{F}\left(p_{1}, p_{2}, \cos \theta\right)+f_{G}\left(p_{1}, p_{2}, \cos \theta\right) .
$$

It may be expected that for excess energies of the order of tens of $\mathrm{eV}$ the dominant contributions to the transition amplitude will be given by the lowest individual electron orbital angular momenta. Indeed, as can be seen from our numerical results, presented in the following two sections, only $s, p, d$, and $f$ waves contribute significantly to the TDCS for excess energies up to $80 \mathrm{eV}$. For this case, the amplitudes $f_{1}$ $\equiv f\left(p_{1}, p_{2}, \cos \theta\right)$ and $f_{2} \equiv f\left(p_{2}, p_{1}, \cos \theta\right)$ in Eq. (3) can be expressed as

$$
\begin{aligned}
& f_{1}=a_{0}+a_{1} P_{2}^{\prime}(\cos \theta)+a_{2} P_{3}^{\prime}(\cos \theta), \\
& f_{2}=b_{0}+b_{1} P_{2}^{\prime}(\cos \theta)+b_{2} P_{3}^{\prime}(\cos \theta),
\end{aligned}
$$

where $P_{2}^{\prime}(x)=3 x, P_{3}^{\prime}(x)=\left(15 x^{2}-3\right) / 2$, and $a_{i}, b_{i}$ are complex coefficients,

$$
\begin{aligned}
a_{0}=- & {\left[D_{F}^{(10)}\left(p_{1}, p_{2}\right)+D_{F}^{(01)}\left(p_{2}, p_{1}\right)\right]-\left[D_{F}^{(12)}\left(p_{1}, p_{2}\right)\right.} \\
& \left.+D_{F}^{(21)}\left(p_{2}, p_{1}\right)\right], \\
a_{1}=+ & {\left[D_{F}^{(21)}\left(p_{1}, p_{2}\right)+D_{F}^{(12)}\left(p_{2}, p_{1}\right)\right]+\left[D_{F}^{(32)}\left(p_{2}, p_{1}\right)\right.} \\
+ & \left.D_{F}^{(23)}\left(p_{1}, p_{2}\right)\right], \\
& a_{2}=-\left[D_{F}^{(32)}\left(p_{1}, p_{2}\right)+D_{F}^{(23)}\left(p_{2}, p_{1}\right)\right],
\end{aligned}
$$

and the coefficients $b_{i}$ are obtained from $a_{i}$ by exchange of $p_{1}$ and $p_{2}$. In Sec. III we present results for the TDCS obtained using this parametrization along with a table of coefficients $a_{i}$ and $b_{i}$ for various excess energies and energy sharings. We also compare the TDCS results obtained using $s, p, d$, and $f$ waves with those obtained from our calculations that account for all angular momenta, which are described in the following section.

\section{Exact account of all individual electron angular momenta}

At high excess energies (of the order of hundreds of eV), the $a b$ initio parametrization (4) for the angular dependence of the polarization-invariant amplitude $f\left(p, p^{\prime}, \cos \theta\right.$ ) (as well as the similar parametrization (25) of the LOPT amplitude) becomes ineffective, since it becomes necessary to account for a large number of individual electron orbital angular momenta. Although the numerical technique described in Appendix A allows one to calculate the dynamical parameters $D_{F, G}^{(l, l \pm 1)}\left(p_{1}, p_{2}\right)$ up to high values of $l$, a closed form for the polarization-invariant amplitude is useful for the analysis of the TDCS at high excess energies. Also, an expression for $f$ in closed form allows one to estimate the contributions of high orbital angular momenta.

The key idea of the approach, which allows one to account exactly for all individual electron orbital angular momenta, is to avoid the use of the partial-wave expansions in Eqs. (7)-(9) by employing instead of Eq. (16) a closed form for the continuum Coulomb wave functions,

$$
\psi_{\mathbf{p}}^{(-)}=(2 \pi)^{-3 / 2} A_{p}^{(-)} e^{i \mathbf{p} \cdot \mathbf{r}} \Phi(-i \eta, 1 ;-i(p r+\mathbf{p} \cdot \mathbf{r})),
$$

where $A_{p}^{(-)}=\exp (\pi \eta / 2) \Gamma(1+i \eta)$, and employing instead of Eq. (17) the integral expression for the CGF in parabolic coordinates $[50,51]$,

$$
\begin{aligned}
G_{E}(\xi, & \left.\eta, \phi ; \xi^{\prime}, \eta^{\prime}, \phi^{\prime}\right) \\
= & \frac{1}{\pi \nu} \int_{0}^{1} d x \frac{x^{-Z \nu}}{(1-x)^{2}} \exp \left(-\frac{\xi+\xi^{\prime}+\eta+\eta^{\prime}}{2 \nu} \frac{1+x}{1-x}\right) \\
& \times \sum_{m} I_{m}\left(\frac{2 \sqrt{x \xi \xi^{\prime}}}{\nu(1-x)}\right) I_{m}\left(\frac{2 \sqrt{x \eta \eta^{\prime}}}{\nu(1-x)}\right) \\
& \times e^{i m\left(\varphi-\varphi^{\prime}\right)} .
\end{aligned}
$$


For $\varphi_{0}(r)$ given by Eq. (10), the potential $U_{\mathbf{p}}(\mathbf{r})$ in Eq. (9) may be transformed into the following form (for $Z_{s}=Z$, a similar expression is given in Ref. [52]):

$$
U_{\mathbf{p}}(\mathbf{r})=4 A_{p}^{(-) *}\left(Z_{s} / \pi\right)^{3 / 2} \int \frac{d \mathbf{q}}{q^{2}} v_{\mathbf{p}}(\mathbf{q}) e^{i \mathbf{q} \cdot \mathbf{r}},
$$

where

$$
\begin{aligned}
v_{\mathbf{p}}(\mathbf{q})= & \frac{\left[q^{2}-\left(p+i Z_{s}\right)^{2}\right]^{-1-i \eta}}{\left[Z_{s}^{2}+(\mathbf{p}+\mathbf{q})^{2}\right]^{2-i \eta}}\left(p+i Z_{s}\right) \\
& \times\left[\left(Z_{s}-Z\right)\left(p+i Z_{s}\right)-2 \eta(\mathbf{p} \cdot \mathbf{q})\right] .
\end{aligned}
$$

This expression is obtained through the use of the integral representation,

$$
\frac{1}{\left|\mathbf{r}-\mathbf{r}^{\prime}\right|}=\frac{1}{2 \pi^{2}} \int \frac{e^{i \mathbf{q} \cdot\left(\mathbf{r}-\mathbf{r}^{\prime}\right)}}{q^{2}} d \mathbf{q},
$$

with subsequent analytical integration over $\mathbf{r}^{\prime}$ in Eq. (9). Since the dependence of $U_{\mathbf{p}}(\mathbf{r})$ on $\mathbf{r}$ in Eq. (31) is simple, all spatial integrals over parabolic coordinates of the vectors $\mathbf{r}$ and $\mathbf{r}^{\prime}$ in Eqs. (7) and (8) can be evaluated analytically (an example of similar calculations is given in Ref. [51]). The result of integration over the azimuthal angle of the vector $\mathbf{q}$, $\varphi_{q}$, can be expressed in terms of the Legendre functions $P_{\mu}(t)$ (see Appendix B). Therefore, the final expressions for the matrix elements $M_{F}$ and $M_{G}$ contain only three remaining integrations: a one-dimensional integral over $x$ [which appears in the integral representation for the CGF in Eq. (30)] and a two-dimensional integral over the components $q$ and $\theta_{q}$ of the vector $\mathbf{q}$. These resulting three-dimensional integrals are to be evaluated numerically. The final expressions for the amplitudes $f_{1} \equiv f\left(p_{1}, p_{2}, \cos \theta\right)$ and $f_{2}$ $\equiv f\left(p_{2}, p_{1}, \cos \theta\right)$, which account for all individual electron orbital angular momenta, may be expressed in terms of two functions, $A\left(p_{1}, p_{2}, \theta\right)$ and $B\left(p_{1}, p_{2}, \theta\right)$, as follows:

$$
\begin{aligned}
& f_{1}=B\left(p_{1}, p_{2}, \theta\right)+A\left(p_{2}, p_{1}, \theta\right)-A\left(p_{1}, p_{2}, \theta\right) \cos \theta, \\
& f_{2}=B\left(p_{2}, p_{1}, \theta\right)+A\left(p_{1}, p_{2}, \theta\right)-A\left(p_{2}, p_{1}, \theta\right) \cos \theta,
\end{aligned}
$$

or, equivalently,

$$
\begin{aligned}
a_{g, u}= & \frac{1}{2}\left\{B\left(p_{1}, p_{2}, \theta\right) \pm B\left(p_{2}, p_{1}, \theta\right)-\left[A\left(p_{1}, p_{2}, \theta\right) \pm A\left(p_{2}, p_{1}, \theta\right)\right]\right. \\
& \times(\cos \theta \mp 1)\} .
\end{aligned}
$$

Explicit integral expressions for $A\left(p_{1}, p_{2}, \theta\right)$ and $B\left(p_{1}, p_{2}, \theta\right)$ are given in Appendix B, where it is also shown that for $\theta$ $=0$ and $\pi$ only the functions $B$ contribute to the amplitudes $f_{1,2}$. We emphasize that the technique described above allows one to calculate the LOPT TDCS over a wide interval of energies without any of the additional approximations that have been used in previous high-energy, LOPT calculations (see, e.g., Refs. [7,44]), such as, e.g., the plane-wave Born approximation for one of photoelectrons, etc.

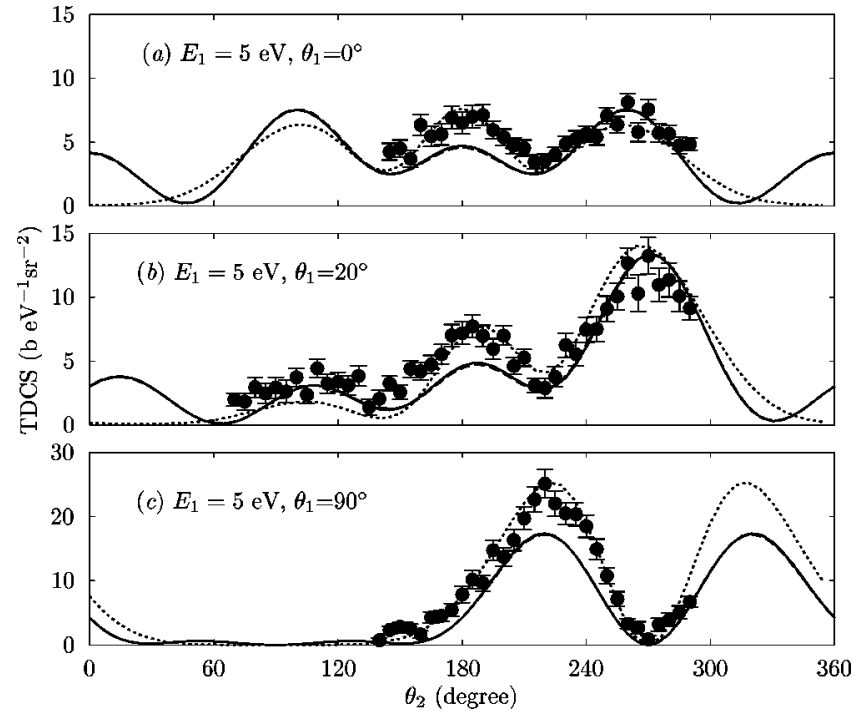

FIG. 3. TDCS for double photoionization of $\mathrm{He}$ at an excess energy of $25 \mathrm{eV}$. Full curves, present LOPT results (with account of FSC and with variational account of electron screening in the He ground state) in which all final-state electron orbital angular momenta are accounted for; dashed curves, present LOPT results but with account of final-state electron $s, p, d$, and $f$ orbital angular momenta only; dotted curves, results of the TDCC calculations of Colgan and Pindzola [45]. The experimental data of Collins et al. [53] have been normalized to the TDCC results at $\theta_{1}=0^{\circ}$ except for the $\theta_{1}=90^{\circ}$ measurements, which were normalized differently, as in Ref. [45]. Note that the dashed curves are virtually indistinguishable from the full curves in this figure.

\section{ANALYSIS OF THE TDCS FOR LINEARLY POLARIZED LIGHT}

In this section we present our results for the TDCS for PDI of He by linearly polarized photons for various excess energies up to $450 \mathrm{eV}$. As described in Sec. II B, we use two substantially different LOPT models to account for electron correlation, one for intermediate excess energies $(25 \mathrm{eV}$, $40 \mathrm{eV}, 60 \mathrm{eV}$, and $80 \mathrm{eV}$ ) and another one for a high excess energy $(450 \mathrm{eV})$. Because the experimental data for these energies are not absolute, we compare our results also with those of accurate $a b$ initio calculations.

\section{A. Intermediate excess energies}

As has been shown in Ref. [40], account of final-state correlation to lowest order, combined with variational account of electron screening in the He ground state, provides TDCSs that are in excellent agreement with available absolute data as well as with accurate ab initio calculations for an excess energy of $20 \mathrm{eV}$. In this section we present our results for the TDCS for excess energies of $25 \mathrm{eV}, 40 \mathrm{eV}, 60 \mathrm{eV}$, and $80 \mathrm{eV}$. We compare our results with available relative experimental data, with results of the time-dependent closecoupling calculations (TDCC) of Colgan and Pindzola [45], and with results of the CCC calculations of Kheifets [46].

Our data presented in Figs. 3-8 were calculated using the model that takes into account FSC to lowest order and uses 

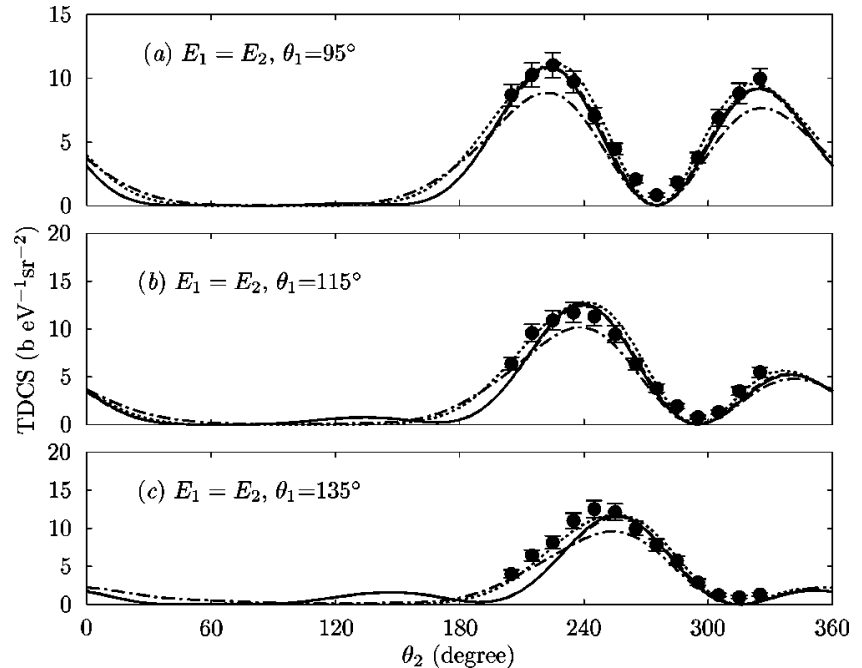

FIG. 4. TDCS for double photoionization of $\mathrm{He}$ at an excess energy of $40 \mathrm{eV}$ for equal energy sharing. Full, dashed, and dotted theoretical curves are defined as in Fig. 3; dot-dashed curves, results of the CCC calculations of Kheifets and Bray [54]. The experimental data of Cvejanovic et al. [54] have been normalized to the TDCC results [45] at $\theta_{1}=95^{\circ}$, as in Ref. [45].

the variational value $Z_{s}=27 / 16$ in the (uncorrelated) groundstate wave function and $Z=2$ in the intermediate and final states. The full curves present results which account for all individual electron orbital angular momenta, while the dashed curves account for $s, p, d$, and $f$ waves only.

In Fig. 3 we compare our results to the experimental data of Collins et al. [53] and to the TDCC calculation of Colgan and Pindzola [45] at an excess energy of $25 \mathrm{eV}$ for an unequal energy-sharing case. As one sees, our results agree in general with both experimental and theoretical results. The

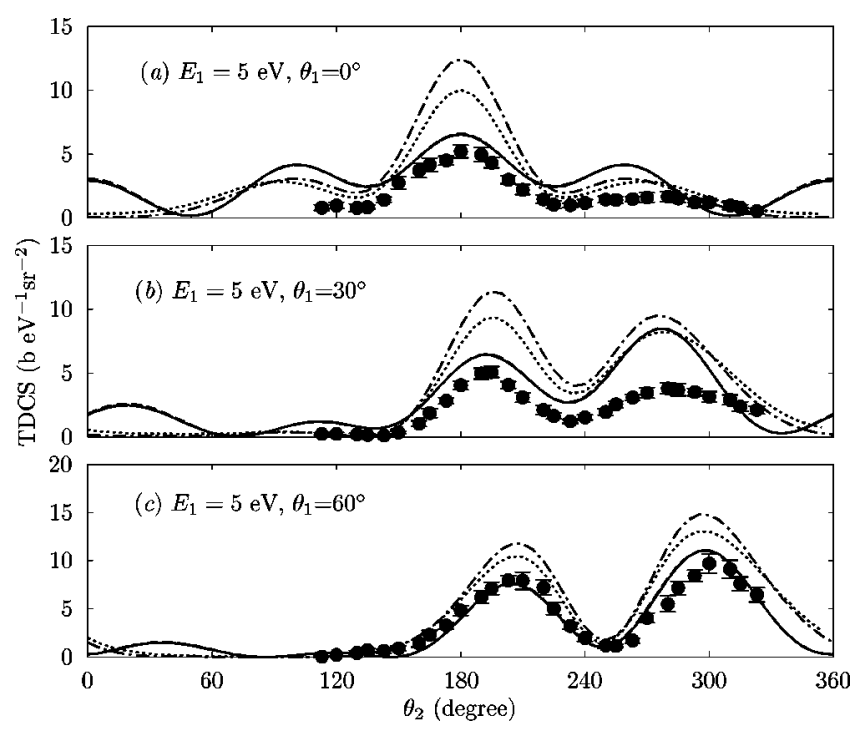

FIG. 5. TDCS for double photoionization of $\mathrm{He}$ at an excess energy of $40 \mathrm{eV}$ for unequal energy sharing: $E_{1}=5 \mathrm{eV}, E_{2}=35 \mathrm{eV}$. Full, dashed, and dotted theoretical curves are defined as in Fig. 3; dot-dashed curves, results of the CCC calculations in Ref. [55]. The experimental data of Bolognesi et al. [55] have been normalized to the CCC results at $\theta_{1}=0$, as described in Ref. [55].

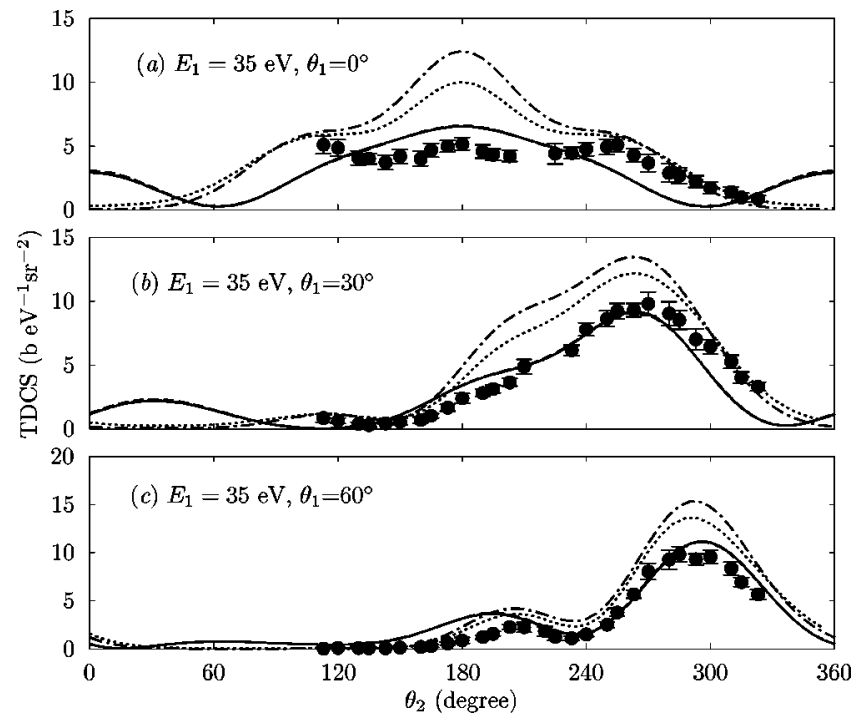

FIG. 6. Same as shown in Fig. 5, but for the case of $E_{1}$ $=35 \mathrm{eV}, E_{1}=5 \mathrm{eV}$.

major discrepancies are similar to those found in Ref. [40]: for the cases of ejection of one electron along (or close to) the photon polarization direction [Figs. 3(a) and 3(b)], there is an unphysical enhancement of the TDCS at small mutual ejection angles $\theta_{12}$. This is due to the inadequacy of a LOPT account of the strong Coulomb repulsion between electrons. In the case of ejection of one electron perpendicular to the photon polarization direction [Fig. 3(c)], the maxima of our predicted TDCSs are smaller than predicted by TDCC theory.

Comparison of our calculated TDCS with the TDCC calculation of Colgan and Pindzola [45] and with the experimental data of Cvejanovic et al. [54] and Bolognesi et al.

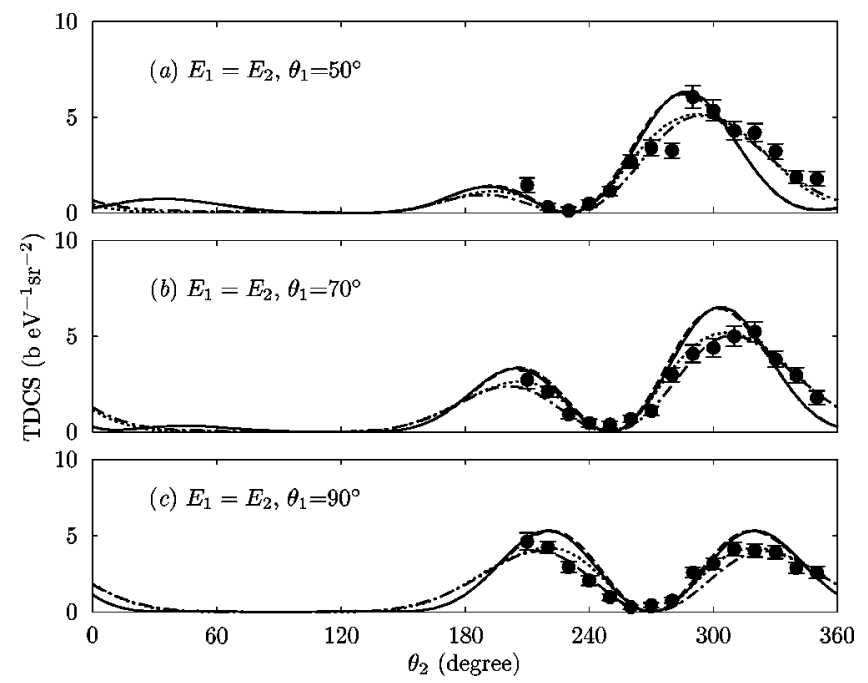

FIG. 7. TDCS for double photoionization of He at an excess energy of $60 \mathrm{eV}$ for equal energy sharing. Full, dashed, and dotted theoretical curves are defined as in Fig. 3; dot-dashed curves, results of the CCC calculations of Kheifets [56]. The experimental data of Dawson et al. [56] have been normalized to the TDCC results of Colgan and Pindzola [45] at $\theta_{1}=90^{\circ}$, as in Ref. [45]. 


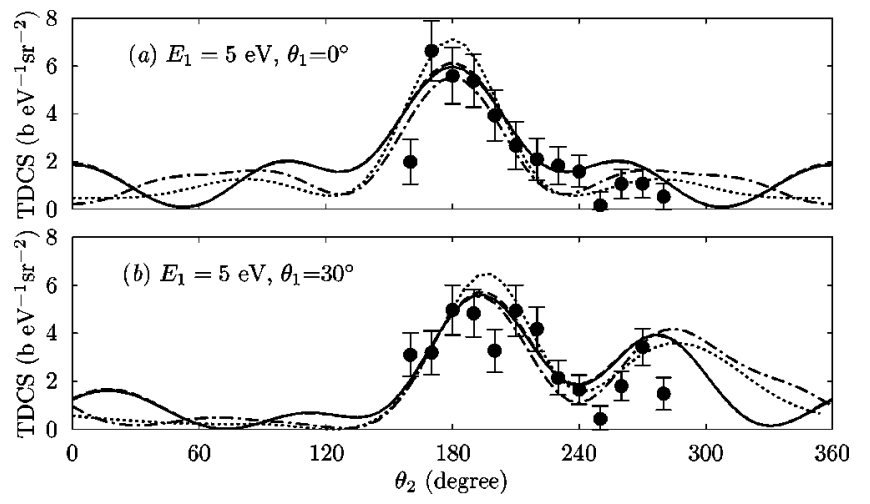

FIG. 8. Same as shown in Fig. 7, but for the case of $E_{1}=5 \mathrm{eV}$, $E_{1}=55 \mathrm{eV}$.

[55] at an excess energy of $40 \mathrm{eV}$ [Figs. 4-6] reveals good agreement, especially for the case of equal energy sharing (Fig. 4). For unequal energy sharing [Figs. 5 and 6], the major disagreements occur near $\theta_{12}=0$. They have the same origin as for the case of an excess energy of $25 \mathrm{eV}$.

For an excess energy of $60 \mathrm{eV}$ [Figs. 7 and 8], reasonable agreement is observed between our LOPT results, the experimental data of Dawson et al. [56] and the TDCC calculation of Colgan and Pindzola [45].

At an excess energy of $80 \mathrm{eV}$ and equal energy sharing (Fig. 9), the discrepancies between predictions of our model and those of the accurate $a b$ initio CCC calculations [46] become more pronounced.

Our results that account for only $s, p, d$, and $f$ final-state electron orbital angular momenta, represented by the dashed curves in Figs. 3-9, were calculated using Eq. (27) for the
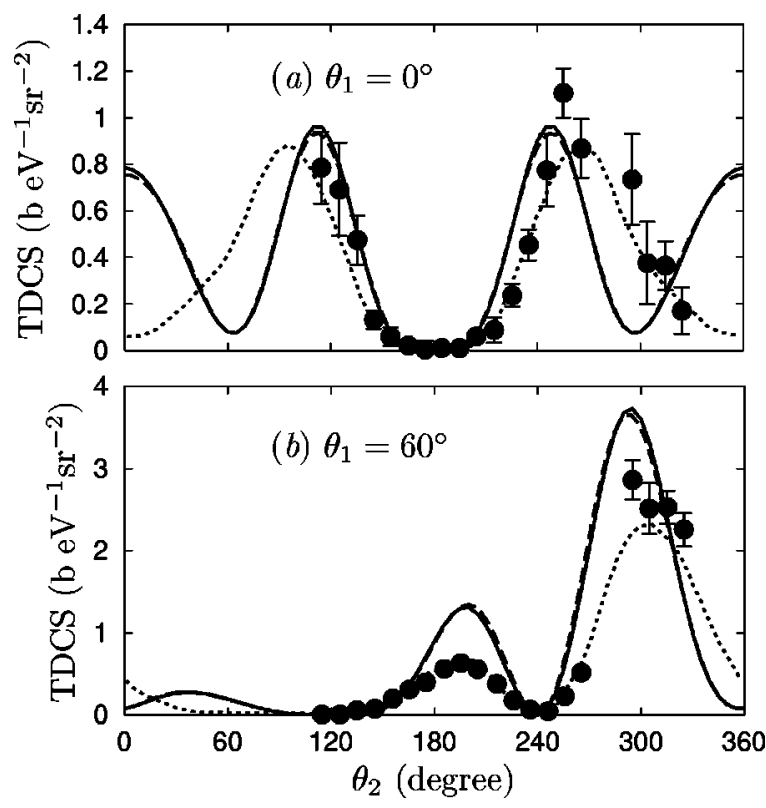

FIG. 9. TDCS for double photoionization of $\mathrm{He}$ at an excess energy of $80 \mathrm{eV}$ for equal energy sharing. Present theoretical results, indicated by the full and dashed curves, are as defined in Fig. 3; dotted curves, results of the CCC calculations in Ref. [46]. The experimental data of Turri et al. [46] have been normalized to the CCC results, as in Ref. [46]. amplitude $f$ in Eq. (26). As one sees, these results are nearly identical to those that account for all angular momenta (represented by the full curves). In Table I we present the coefficients $a_{i}$ and $b_{i}$ that were used in Eq. (27) for all excess energies and energy sharings presented, as well as for a number of intermediate values. Using the data given in the Table I one can easily construct the amplitudes $f_{1}$ and $f_{2}$ as well as the TDCS and the CD parameters for any kinematical situation which corresponds to the particular values of excess energies and energy sharings for which the coefficients $a_{i}$ and $b_{i}$ are given.

\section{B. Physical mechanism of PDI at intermediate energies}

Using our approach, it appears to be possible to identify a distinct physical mechanism for PDI at intermediate excess energies. In our model, which treats FSC to first order, the PDI transition amplitude is given by Eq. (7) and the associated diagram, Fig. 1(a), corresponds to the knock-out mechanism of PDI. The CGF in Eq. (7) is evaluated for a positive energy parameter, $\mathcal{E}=E_{F}$, and may be written using the following spectral representation:

$$
G_{\mathcal{E}}=\sum_{n, l, m} \frac{|n \operatorname{lm}\rangle\langle n \operatorname{lm}|}{E_{n}-\mathcal{E}}+\int d \mathbf{k} \frac{\left|\mathbf{k}^{(-)}\right\rangle\left\langle\mathbf{k}^{(-)}\right|}{k^{2} / 2-\mathcal{E}-i 0} .
$$

Recalling the identity,

$$
\frac{1}{x-i 0}=\mathrm{P} \frac{1}{x}+i \pi \delta(x),
$$

where $\mathrm{P}$ denotes the principal value, the real and imaginary parts of Eq. (36) may be written as

$$
\begin{gathered}
\operatorname{Re} G_{\mathcal{E}}=\sum_{n, l, m} \frac{|n l m\rangle\langle n l m|}{E_{n}-\mathcal{E}}+\mathrm{P} \int d \mathbf{k} \frac{\left|\mathbf{k}^{(-)}\right\rangle\left\langle\mathbf{k}^{(-)}\right|}{k^{2} / 2-\mathcal{E}}, \\
\operatorname{Im} G_{\mathcal{E}}=\pi \int d \Omega_{\mathbf{k}^{\prime}}\left|\mathbf{k}^{\prime(-)}\right\rangle\left\langle\mathbf{k}^{\prime(-)}\right|,
\end{gathered}
$$

where $k^{\prime 2} / 2=\mathcal{E}$. When the real and imaginary parts of the CGF in Eq. (38) are substituted into Eq. (7) for the FSC amplitude, the imaginary part of the CGF gives rise to the so-called "direct" (on-shell) knock-out mechanism for PDI, which has a direct classical interpretation: one electron absorbs the photon, and becomes excited to a continuum state with asymptotic momentum $\mathbf{k}^{\prime}$; then it knocks-out the second electron, and they both leave with asymptotic momenta $\mathbf{p}_{1}$ and $\mathbf{p}_{2}$. Since energy conservation fixes only the energy of the "intermediate" electron [cf. Eq. (38)], the amplitude involves an integration over all directions of $\mathbf{k}^{\prime}$. The PDI amplitude involving the real part of the CGF in Eq. (38) corresponds to the so-called "virtual" knock-out mechanism, which may be considered a quantum counterpart of the classical knock-out process.

Insight into the physical mechanism of the PDI knock-out process can be obtained by comparing the contributions of the real and imaginary parts of the CGF to the transition amplitude (7). One way to do this is to directly evaluate the contribution of the imaginary part of the CGF. In our study, 
TABLE I. Expansion coefficients $a_{i}$ and $b_{i}$ (in atomic units) for the amplitudes $f_{1}$ and $f_{2}$, as defined in Eq. (27), for various excess energies $\left(E_{\text {exc }}\right)$ and energy sharings. In calculating these coefficients, FS correlations are taken into account, a basis set with $Z_{s}=27 / 16$ is used for the He ground-state wave function, and the basis set with $Z=2$ is used for intermediate and final states.

\begin{tabular}{|c|c|c|c|c|c|c|c|}
\hline $\begin{array}{l}E_{e x c} \\
(\mathrm{eV})\end{array}$ & $\begin{array}{c}E_{1} \\
(\mathrm{eV})\end{array}$ & $\begin{array}{c}a_{0} \\
\left(10^{-3} \text { a.u. }\right)\end{array}$ & $\begin{array}{c}a_{1} \\
\left(10^{-3} \text { a.u. }\right)\end{array}$ & $\begin{array}{c}a_{2} \\
\left(10^{-4} \text { a.u. }\right)\end{array}$ & $\begin{array}{c}b_{0} \\
\left(10^{-3} \text { a.u. }\right)\end{array}$ & $\begin{array}{c}b_{1} \\
\left(10^{-3} \text { a.u. }\right)\end{array}$ & $\begin{array}{c}b_{2} \\
\left(10^{-4} \text { a.u. }\right)\end{array}$ \\
\hline \multirow[t]{3}{*}{9} & 4.5 & $-18.29+11.91 i$ & $8.199-5.939 i$ & $-1.115+6.720 i$ & $-18.29+11.91 i$ & $8.199-5.939 i$ & $-1.115+6.720 i$ \\
\hline & 6 & $-10.00+19.29 i$ & $5.165-9.446 i$ & $2.132+7.064 i$ & $-10.52+20.58 i$ & $3.302-9.493 i$ & $2.994+5.840 i$ \\
\hline & 8 & $-14.81-13.41 i$ & $13.33+2.802 i$ & $-7.288+6.034 i$ & $-29.08-2.594 i$ & $11.33-2.625 i$ & $-2.669+6.604 i$ \\
\hline \multirow[t]{2}{*}{15} & 7.5 & $-2.895-14.68 i$ & $1.215+7.299 i$ & $-3.993-4.311 i$ & $-2.895-14.68 i$ & $1.215+7.299 i$ & $-3.993-4.311 i$ \\
\hline & 12 & $-15.20+1.501 i$ & $8.946+0.551 i$ & $-5.897+4.120 i$ & $-17.62+2.231 i$ & $7.097-2.203 i$ & $-3.059+4.497 i$ \\
\hline \multirow[t]{4}{*}{20} & 10 & $6.616-9.709 i$ & $-3.779+4.765 i$ & $1.049-5.302 i$ & $6.616-9.709 i$ & $-3.779+4.765 i$ & $1.049-5.302 i$ \\
\hline & 14.5 & $0.807-11.53 i$ & $-1.665+6.744 i$ & $-1.790-5.929 i$ & $0.476-13.09 i$ & $0.204+5.844 i$ & $-2.551-4.199 i$ \\
\hline & 17 & $-11.82-2.878 i$ & $7.199+3.564 i$ & $-6.954+1.146 i$ & $-14.74-2.809 i$ & $6.132-0.058 i$ & $-3.953+2.777 i$ \\
\hline & 17.5 & $-11.61+4.473 i$ & $8.316-1.144 i$ & $-5.179+5.189 i$ & $-14.29+6.405 i$ & $4.992-3.816 i$ & $-1.363+4.671 i$ \\
\hline 25 & 20 & $1.427-9.382 i$ & $-2.382+5.987 i$ & $-0.829-6.120 i$ & $1.031-11.45 i$ & $0.110+4.931 i$ & $-2.049-3.862 i$ \\
\hline \multirow[t]{3}{*}{40} & 20 & $5.484+1.924 i$ & $-3.043-1.644 i$ & $4.082+1.028 i$ & $5.484+1.924 i$ & $-3.043-1.644 i$ & $4.082+1.028 i$ \\
\hline & 30 & $5.580-0.049 i$ & $-4.084-0.982 i$ & $4.902-0.539 i$ & $6.680-0.694 i$ & $-3.134+0.321 i$ & $3.319-1.238 i$ \\
\hline & 35 & $2.973-4.971 i$ & $-3.680+3.446 i$ & $1.959-5.214 i$ & $3.054-7.298 i$ & $-0.781+3.133 i$ & $-0.349-3.248 i$ \\
\hline \multirow[t]{2}{*}{50} & 25 & $3.577+2.626 i$ & $-1.912-2.015 i$ & $3.061+2.201 i$ & $3.577+2.626 i$ & $-1.912-2.015 i$ & $3.061+2.201 i$ \\
\hline & 45 & $2.831-3.386 i$ & $-3.614+2.452 i$ & $2.629-4.387 i$ & $3.045-5.634 i$ & $-0.818+2.415 i$ & $0.074-2.735 i$ \\
\hline \multirow[t]{3}{*}{60} & 30 & $2.325+2.611 i$ & $-1.167-1.964 i$ & $2.130+2.636 i$ & $2.325+2.611 i$ & $-1.167-1.964 i$ & $2.130+2.636 i$ \\
\hline & 50 & $3.180+0.994 i$ & $-2.757-1.674 i$ & $2.443+0.262 i$ & $4.446+0.448 i$ & $-1.813-0.056 i$ & $3.111-0.371 i$ \\
\hline & 55 & $2.542-2.362 i$ & $-3.380+1.783 i$ & $2.908-3.651 i$ & $2.816-4.474 i$ & $-0.766+1.913 i$ & $0.284-2.298 i$ \\
\hline \multirow[t]{2}{*}{70} & 35 & $1.520+2.377 i$ & $-0.688-1.784 i$ & $1.405+2.715 i$ & $1.520+2.377 i$ & $-0.688-1.784 i$ & $1.405+2.715 i$ \\
\hline & 65 & $2.241-1.675 i$ & $-3.102+1.318 i$ & $2.982-3.025 i$ & $2.538-3.633 i$ & $-0.692+1.548 i$ & $0.386-1.941 i$ \\
\hline \multirow[t]{2}{*}{80} & 40 & $0.996+2.096 i$ & $-0.379-1.577 i$ & $0.866+2.626 i$ & $0.996+2.096 i$ & $-0.379-1.577 i$ & $0.866+2.626 i$ \\
\hline & 70 & $1.937+1.072 i$ & $-1.855-1.665 i$ & $3.148+1.564 i$ & $3.114+0.620 i$ & $-1.338-0.199 i$ & $1.824+0.136 i$ \\
\hline
\end{tabular}

however, it is more convenient to use the results already obtained for the partial-wave amplitudes $M_{F}^{\left(l_{1} l_{2}\right)}$ in Eq. (21). If one uses the multipole expansions for the continuum states, the CGF may be rewritten in terms of eigenstates of energy and orbital angular momentum, $|E l m\rangle$, to yield

$$
\begin{gathered}
\operatorname{Re} G_{\mathcal{E}}=\sum_{l, m}\left(\sum_{n} \frac{|n l m\rangle\langle n l m|}{E_{n}-\mathcal{E}}+\mathrm{P} \int d E \frac{|E \operatorname{lm}\rangle\langle E \ln |}{E-\mathcal{E}}\right), \\
\operatorname{Im} G_{\mathcal{E}}=\pi \sum_{l, m}|\mathcal{E} \operatorname{lm}\rangle\langle\mathcal{E} \operatorname{lm}|
\end{gathered}
$$

The contributions of the real and imaginary parts of the CGF to each term $M_{F}^{\left(l_{1} l_{2}\right)}$ can now be extracted easily; they are given by the real and imaginary parts of the radial matrix elements $\mathcal{R}_{F}^{\left(l_{1} l_{2}\right)}$ [see Eqs. (A2) and (A14) in Appendix A], which will be discussed in detail in Sec. V.

Our results for the TDCS, in which we account either for real (dashed curves) or imaginary (dotted curves) parts of the $\mathrm{CGF}$, are presented for the excess energies of $25 \mathrm{eV}$ and $60 \mathrm{eV}$ in Fig. 10. As one sees, the real part alone gives a correct qualitative description of the TDCS for intermediate and large mutual ejection angles for all excess energies presented, while the imaginary part of the CGF gives only a small correction. Our analysis of other kinematical situations for excess energies up to $60 \mathrm{eV}$ (not presented in Fig. 10) reveals similar findings. We conclude therefore, that the dominant physical mechanism for PDI at intermediate excess energies and intermediate and large mutual ejection angles is the virtual (off-shell) knock-out process. This conclusion appears to be gauge invariant: a similar analysis of the amplitudes calculated using the length form for the dipole transition operator (not presented here) shows that for intermediate energies the relative contribution of the on-shell knock-out mechanism in the length gauge is even smaller than that in the velocity gauge. Note that, as follows from the equations corresponding to Eqs. (11) and (13) for the partial-wave amplitudes, $M_{F}^{\left(l_{1}, l_{2}\right)}$, the on-shell knock-out contribution (given by the imaginary part of the CGF) is gauge independent, provided a single basis set is used in the calculation [because the quantity $\Delta M^{\left(l_{1}, l_{2}\right)}$ in Eqs. (11)-(13) is real, apart from the multiplicative Coulomb phase-shift factors that appear in each term of Eq. (11)]. Although we use two different basis sets in our model, the absolute values of our on-shell knockout mechanism contributions are of the same magnitude in both length and velocity gauges, while the contributions of the off-shell knock-out mechanism are two to three times larger in the length gauge. Thus, although the shapes of the length-gauge TDCSs are very similar to the velocity-gauge ones, the quantitative discrepancies between the lengthgauge TDCSs and the absolute experimental data are much greater [40]. 

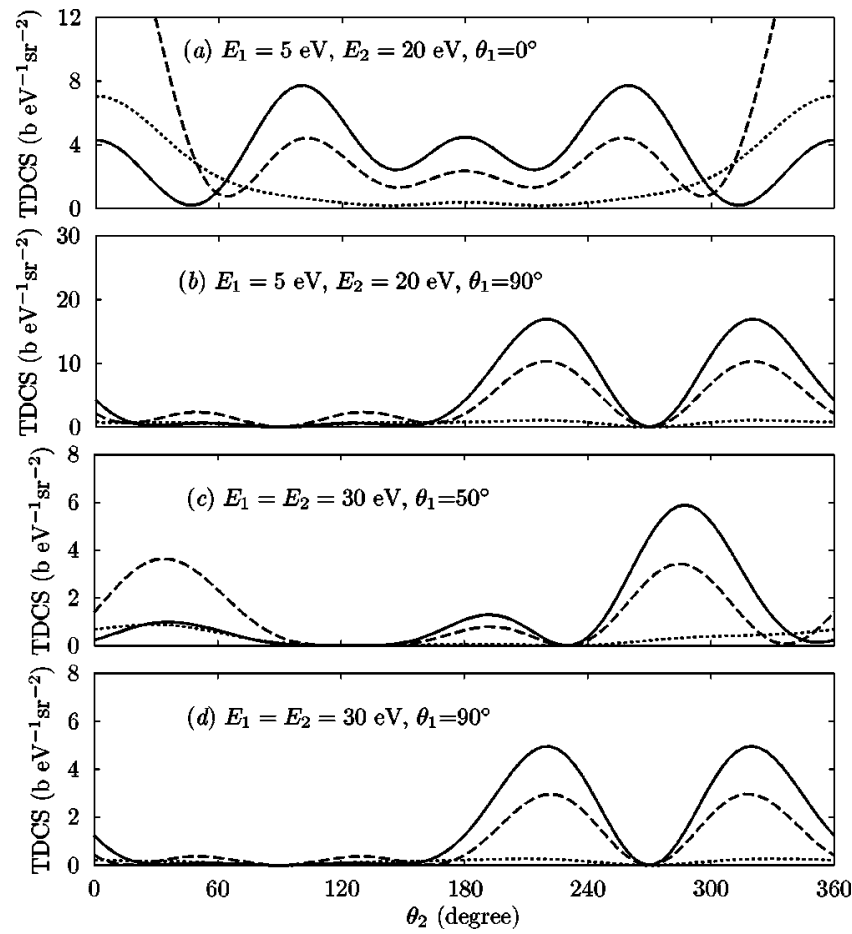

FIG. 10. TDCS with account of $s, p, d$, and $f$ final-state electron orbital angular momenta for an excess energies of $25 \mathrm{eV}$ (a) and (b) and $60 \mathrm{eV}$ (c) and (d). Full curves, present exact results (using the full CGF, $G_{E_{F}}$ ); dashed curves, "virtual" knock-out only (i.e., using $\operatorname{Re} G_{E_{F}}$ ); dotted curves, "direct" knock-out only (i.e., using $\operatorname{Im} G_{E_{F}}$ ). [Note: CGF denotes the Coulomb Green function; see Eqs. (36) and (38).]

\section{High excess energies}

The results of several of our calculations show that our first model, which accounts for electron correlation only in the final state, fails to describe the TDCS correctly at high excess energies (i.e., for excess energies of a few hundred $\mathrm{eV})$. We find that an account of electron correlations in both final and ground states is necessary at such excess energies.

In Fig. 11 we present results using our LOPT approach with account of both FS and GS correlations, in which the bare charge $Z=2$ is used in all unperturbed one-electron basis states. Because we include all diagrams of the given (i.e., the first) PT order, results of this calculation are gauge independent. We compare our results with the relative experimental data of Knapp et al. [36] and with the CCC calculation results of Kheifets and Bray [36] at an excess energy of $450 \mathrm{eV}$.

The plots given in Figs. 11(a) and 11(b) present the TDCS for a strongly asymmetric energy-sharing regime. In the case when the slow electron $(2 \mathrm{eV})$ is ejected along the photon polarization direction, shown in Fig. 11(a), the fast electron $(448 \mathrm{eV})$ exhibits a $p$-wave angular distribution, which corresponds to the single ionization limit. In the case when the fast electron is ejected along the photon polarization direction, shown in Fig. 11(b), the angular distribution of the slow electron shows clear evidence of shake-off into the $s$-wave continuum. As one sees, there are clear discrepancies between our calculated TDCS and the CCC results for this strongly asymmetric energy-sharing case.
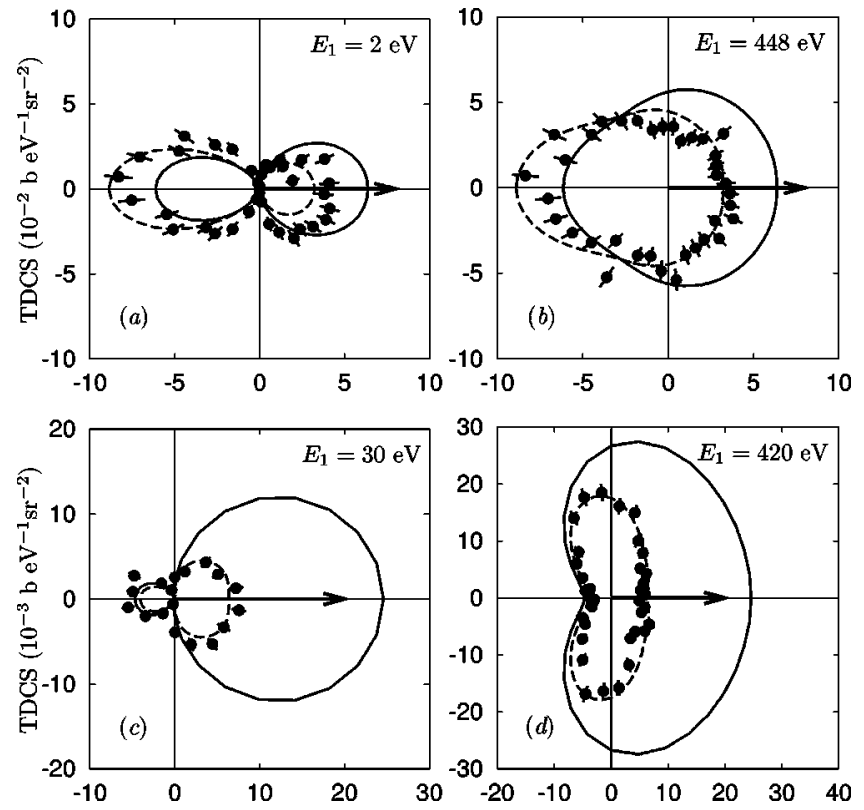

FIG. 11. TDCS for an excess energy of $450 \mathrm{eV}$. The electron having energy $E_{1}$ is emitted along the photon polarization direction, indicated by the arrows. Full curves, gauge-invariant $\operatorname{LOPT}\left(Z_{s}=2\right)$ results; dashed curves, CCC results from Knapp et al. [36]. The experimental data of Knapp et al. [36] have been normalized to the CCC results, as in Ref. [36].

The TDCSs presented in Figs. 11(c) and 11(d) correspond to another case of unequal (but not so strongly asymmetric) energy sharing. For this kinematical situation, the LOPTcalculated TDCS is comprised of a strongly destructive interference between the FS and the GS correlation amplitudes. One sees that there is agreement of our results with the CCC results at large mutual ejection angles $\left[\left|\theta_{12}\right|>90^{\circ}\right.$ in Fig. 11(c) and $\left|\theta_{12}\right|>120^{\circ}$ in Fig. 11(d)] and disagreement for small mutual angles.

In summary, our LOPT results confirm that while the PDI is dominated by the knock-out mechanism at intermediate excess energies, for high excess energies (of a few hundred $\mathrm{eV}$ ) electron correlation in the ground state becomes at least of equal importance.

\section{Contributions of high angular momenta}

Theoretical treatments for high-energy PDI must necessarily account for a large number of electron orbital angular momenta. Therefore it is of interest to study how the contributions of high angular momenta depend upon excess energy.

It can be seen from Figs. 3-9, where we compare results that account for $s p, p d$, and $d f$ final-state two-electron channels with results that account exactly for all angular momenta, for excess energies of tens of $\mathrm{eV}$ the relative contribution of high angular momenta (i.e., $l>3$ ) is almost negligible. The discrepancies between these two descriptions are seen to become noticeable only at an excess energy of $80 \mathrm{eV}$ (cf. Fig. 9).

In contrast, at higher excess energy (of the order of hundreds of eV), especially for not very asymmetric energy shar- 
ing, the contribution of higher angular momenta $(l>3)$ is comparable to that of the $s p, p d$, and $d f$ channels. Our calculations of the $s p, p d$, and $d f$ partial-wave amplitudes at high excess energy allow us to find the relative contributions of each channel. For the cases of equal energy sharing and an excess energy of $E_{\text {exc }}=200 \mathrm{eV}$, we find that $\left|a_{0}\right| /\left|a_{1}\right|=1.5$ and $\left|a_{1}\right| /\left|a_{2}\right|=4.9$, for $E_{e x c}=300 \mathrm{eV}, \quad\left|a_{0}\right| /\left|a_{1}\right|=1.4$ and $\left|a_{1}\right| /\left|a_{2}\right|=4.0$, for $E_{\text {exc }}=450 \mathrm{eV},\left|a_{0}\right| /\left|a_{1}\right|=1.3$ and $\left|a_{1}\right| /\left|a_{2}\right|$ $=3.5$. Thus, the relative contributions of higher angular momenta increase with increasing excess energy. For the case of a very asymmetric energy sharing, however, $E_{1}=448 \mathrm{eV}$ and $E_{2}=2 \mathrm{eV},\left|a_{0}\right| /\left|a_{1}\right|=1.2$ and $\left|a_{1}\right| /\left|a_{2}\right|=7.6$. Therefore, in this case the contribution of the $d f$ channel is much less than that for the symmetric energy-sharing case.

\section{ANALYSIS OF THE TDCS AND CIRCULAR DICHROISM FOR THE CASE OF CIRCULARLY POLARIZED LIGHT}

In this section we use our LOPT approach to elucidate the dominant physical mechanism of PDI which is responsible for the circular dichroism effect, and to analyze the general properties of the $\mathrm{CD}$ parameter. By introducing the notation $\sigma(\xi) \equiv \sigma_{T D C S}(\xi)$, where $\xi$ is the degree of circular polarization of the incident light, the absolute and normalized CD parameters $\Delta_{c d}$ and $\delta_{c d}$ are defined as

$$
\Delta_{c d}=\sigma(+1)-\sigma(-1), \quad \delta_{c d}=\frac{\sigma(+1)-\sigma(-1)}{\sigma(+1)+\sigma(-1)} .
$$

We carry out our analysis for the geometrical arrangement used in the experiment of Ref. [31], in which the electrons are ejected in the plane perpendicular to the photon wave vector. We denote the mutual ejection angle between the two electrons by $\theta_{12}$. Therefore, the explicit expressions for the CD parameters, which follow from Eq. (5), are

$$
\begin{gathered}
\Delta_{c d}=2 C_{0} \operatorname{Im}\left\{f_{1} f_{2}^{*}\right\} \sin \theta_{12}, \\
\delta_{c d}=\frac{2 \operatorname{Im}\left\{f_{1} f_{2}^{*}\right\} \sin \theta_{12}}{\left|f_{1}\right|^{2}+\left|f_{2}\right|^{2}+2 \operatorname{Re}\left\{f_{1} f_{2}^{*}\right\} \cos \theta_{12}} .
\end{gathered}
$$

In Fig. 12 we present our results for both the TDCS and the CD effect parameter in Eq. (42) for the case of an excess energy of $20 \mathrm{eV}$ and for several energy sharings for which absolute experimental data are available [31]. As can be seen from Figs. 12(a)-12(d), our predictions for the TDCS (full curves in bold), are in reasonable agreement with experiment for all energy sharings considered. Excellent agreement with experiment is found for the circular dichroism parameter (full curves in bold), presented in Figs. 12(e)-12(h). Analysis of our results for this case enables us to elucidate the physical mechanisms of the CD effect, which is the subject of Sec. IV A below.

At the excess energy of $60 \mathrm{eV}$, our predictions for the dichroism parameter $\Delta_{c d}$ in Eq. (41) agree in general with those of the HRM-SOW calculations of Ref. [37], as shown in Fig. 13. A striking discrepancy, however, is that our results predict two nongeometrical zeros: one in the vicinity of $\theta_{12}$ $=90^{\circ}$ and the second one near $\theta_{12}=40^{\circ}$, while according to
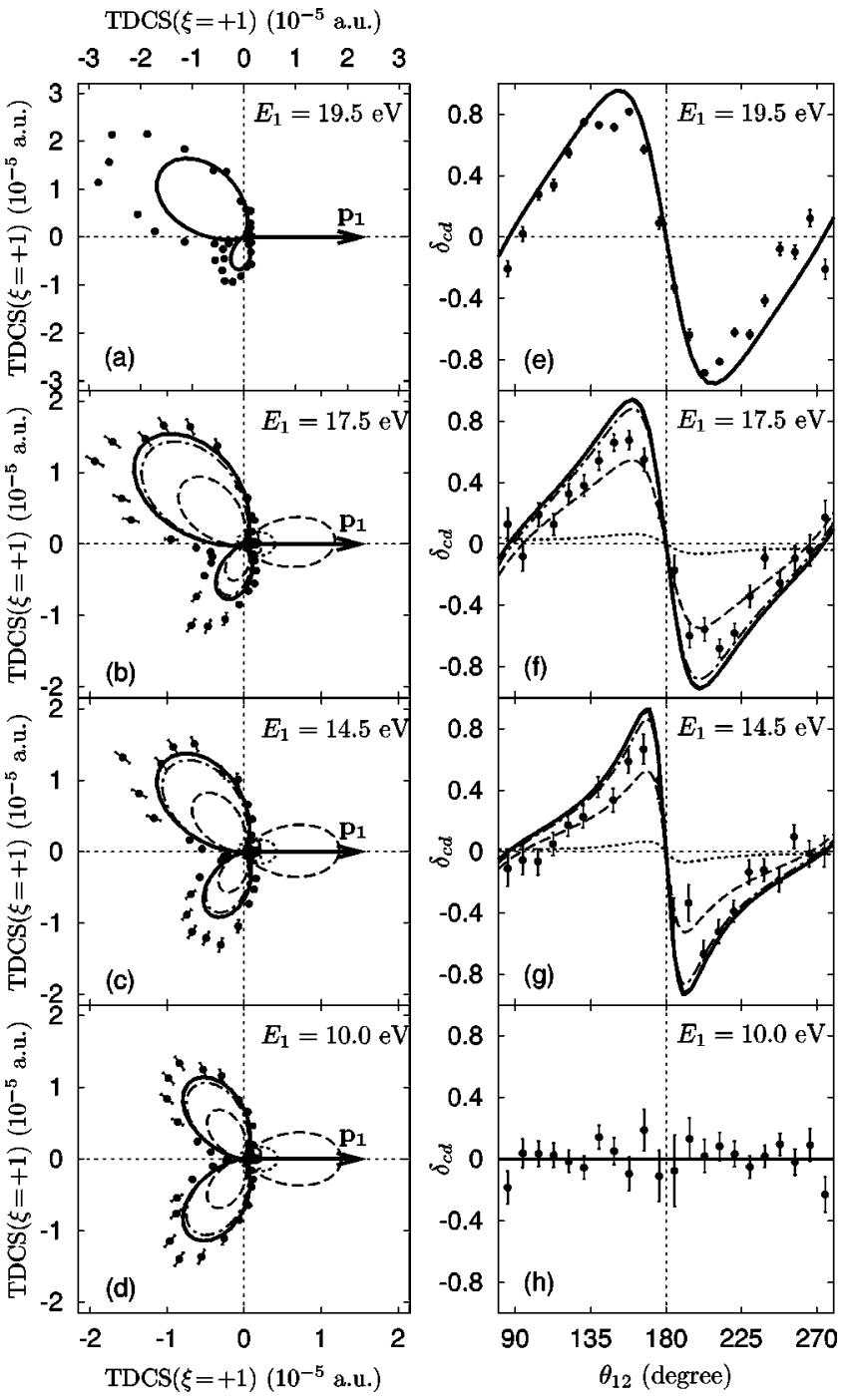

FIG. 12. TDCS (for $\xi=+1$ ) and the normalized circular dichroism parameter $\delta_{c d}$ [cf. Eq. (40)] for PDI of He at an excess energy of $20 \mathrm{eV}$. FS correlation is taken to first order, the effective charge $Z_{s}=27 / 16$ is used in the He ground state, and $Z=2$ is used in the intermediate and final states. All curves in bold, present results including all angular momenta; all curves of regular thickness, account of only $s, p, d$, and $f$ waves; full curves, account of both Re and Im parts of the CGF, $G_{E_{F}}$; dashed curves, Re $G_{E_{F}}$ only; dotted curves, Im $G_{E_{F}}$ only; dot-dashed curves, $\operatorname{Re} G_{E_{F}}$ with account of interference terms containing $\operatorname{Im} G_{E_{F}}$. Experimental data, Achler et al. [31]. [Note: CGF denotes the Coulomb Green function; see Eqs. (36) and (38).]

the predictions of Ref. [37] $\Delta_{c d}$ is zero only for $\theta_{12}=0$ and $\theta_{12}=180^{\circ}$, which are the so-called "geometrical zeros." In Sec. IV B. below we analyze in detail the occurrence of these nongeometrical zeros of the $\mathrm{CD}$ parameters.

\section{A. Physical mechanism of the CD effect}

As pointed out in Sec. III, the dominant mechanism of PDI by linearly polarized light at intermediate energies is the virtual knock-out process. In Fig. 12 we present the separate contributions of the real (dashed curves) and the imaginary 


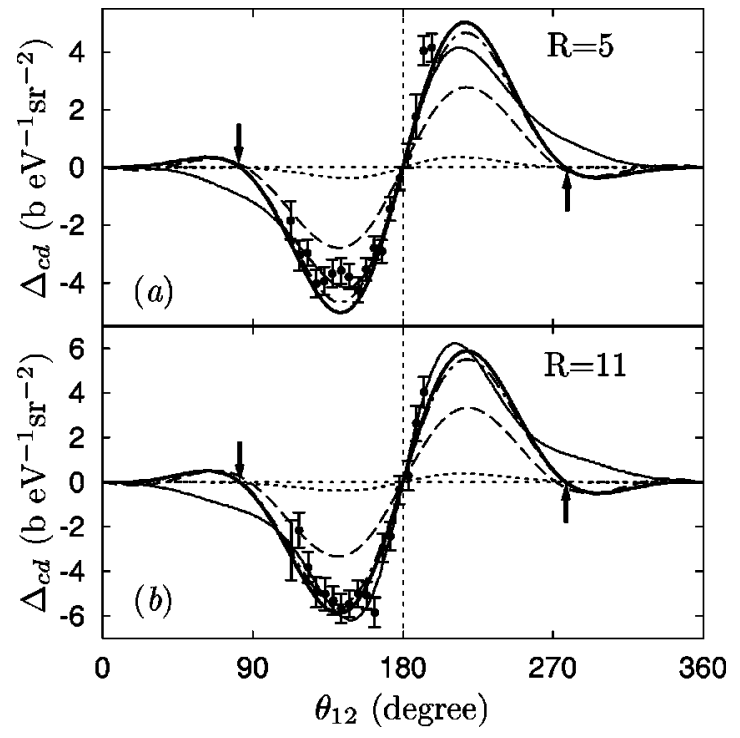

FIG. 13. Circular dichroism parameter $\Delta_{c d}$ for PDI of He at an excess energy of $60 \mathrm{eV}$, for two different values of $R=E_{1} / E_{2}$. Bold solid curves, present LOPT results with account of all angular momenta; dashed, dotted, and dot-dashed curves, present LOPT results with account of only $s, p, d$, and $f$ waves. Dashed curves, account of $\operatorname{Re} G_{E_{F}}$ only; dotted curves, account of $\operatorname{Im} G_{E_{F}}$ only; dot-dashed curves, $\operatorname{Re} G_{E_{F}}$ with account of interference terms containing Im $G_{E_{F}}$. Regular solid curves, results of the HRM-SOW calculations in Ref. [57]. Experimental data are from Ref. [57]. The positions of the nongeometrical CD zero points are indicated by arrows. [Note: $G_{E_{F}}$ denotes the Coulomb Green function; see Eqs. (36) and (38).]

(dotted curves) parts of the CGF to the TDCS and to the $\delta_{c d}$ parameter in Eq. (42) together with the results which account for both contributions (full curves in bold). As one can see from this figure, PDI by circularly polarized photons is also dominated by the virtual knock-out process. This fact allows us to identify the origin of the CD effect in PDI for intermediate excess energies.

As shown by the general parametrization of the TDCS in Eq. (5), the CD effect originates from an interference between the amplitudes $f_{1}$ and $f_{2}$ and is nonzero only for complex amplitudes $f_{1}$ and/or $f_{2}$. If one neglects the contribution of the imaginary part of the CGF to the PDI transition amplitude (7), the transition amplitude remains nevertheless complex (and thus non-Hermitian) and predicts a CD parameter that is in agreement with experimental data. This complexity of the amplitude stems from the complex multiplicative factors $\exp \left\{i\left(\delta_{l_{1}}\left(p_{1}\right)+\delta_{l_{2}}\left(p_{2}\right)\right)\right\}$, which are due to the nonzero Coulomb phase shifts, $\delta_{l}(p)$. Note that in the planewave Born approximation for the one-particle continuum wave functions $\psi_{\mathbf{p}}^{(-)}(\mathbf{r})$, the amplitude is real (assuming the imaginary part of the corresponding Green function is negligible) and thus the CD effect vanishes. However, if one takes into account even the first Born correction (due to the Coulomb potential), then the transition amplitude becomes complex and the CD effect becomes nonzero. Account of the first Born correction corresponds to account of elastic scattering of the photoelectrons from the nucleus. Indeed, a simple analysis shows that the imaginary part of this first-Born PDI amplitude factorizes into a product of the plane-wave PDI amplitude and the amplitude for elastic Coulomb scattering of one of the electrons. Therefore, the fact that the transition amplitude is complex primarily due to the Coulomb phase shifts (owing to the smallness of the contribution of the imaginary part of the CGF to the FSC amplitude) allows us to draw the conclusion that the $\mathrm{CD}$ effect originates from the interference of two two-electron phase-locked wave packets: one, consisting of (freely-propagating) plane waves and the other that is elastically scattered from the nucleus.

\section{B. Nongeometrical zeros of the CD parameters}

The origin of nongeometrical (i.e., dynamical) zeros of the CD parameters has become a longstanding question since the general structure of the CD parameters was first analyzed in Refs. $[15,16]$. The geometrical zeros of the dichroism parameter $\Delta_{c d}$ are due to zeros of $\sin \theta_{12}$ in Eq. (41) and occur at $\theta_{12}=0$ and $\theta_{12}=180^{\circ}$. The existence of nongeometrical (or dynamical) zeros of $\Delta_{c d}$, originating from zeros of the dynamical factor $\operatorname{Im}\left\{f_{1} f_{2}^{*}\right\}$, were predicted [16]. In a numerical example in Ref. [16], the position of a zero point of the CD parameters as a function of the coordinates $\left(E_{1}, E_{2}, \theta_{12}\right)$ was estimated to be at $E_{1}=31 \mathrm{eV}, E_{2}=3 \mathrm{eV}$, and $\theta_{12}=90^{\circ}$. Nongeometrical zero points of the $\mathrm{CD}$ parameters have also been both predicted by recent $\mathrm{CCC}$ calculations and measured experimentally (for an excess energy of $20 \mathrm{eV}$ ), in the vicinity of $\theta_{12}=90^{\circ}$ [31]. However, dynamical zeros for the CD parameter were not found in all studies of the effect. As mentioned above, dynamical zeros were neither predicted by the HRM-SOW theory nor observed experimentally for an excess energy of $60 \mathrm{eV}$ [57].

In contrast to the results of Ref. [57], we find that for an excess energy of $60 \mathrm{eV}$ (for energy sharings of $R \equiv E_{1} / E_{2}$ $=5$ and $R=11$ ) there are two nongeometrical zero points in the CD parameter in the vicinities of $\theta_{12}= \pm 90^{\circ}$, indicated by the arrows in Fig. 13. This case is thus similar to that for an excess energy of $20 \mathrm{eV}$, shown in Fig. 12. Our theory also predicts two additional nongeometrical zero points in the vicinities of $\theta_{12}= \pm 40^{\circ}$, but these might be an artefact of our LOPT approach, which does not provide accurate values for the TDCS for small mutual ejection angles. An independent $a b$ initio calculation would be necessary to either confirm or reject these latter predictions.

Use of our LOPT approach together with the parametrization of the amplitudes $f_{1}$ and $f_{2}$ in Eq. (27), permits us to gain some insight into the appearance of two of the nongeometrical zeros of the CD parameter, namely, the ones which occur in the vicinity of $\theta_{12}= \pm 90^{\circ}$. By substituting the amplitudes $f_{1}$ and $f_{2}$ given by Eq. (27) into the expression for $\Delta_{c d}$ in Eq. (41), $\Delta_{c d}$ takes the following form:

$$
\Delta_{c d}=c_{0}+c_{1} \cos \theta_{12}+c_{2} \cos ^{2} \theta_{12}+c_{3} \cos ^{3} \theta_{12}+c_{4} \cos ^{4} \theta_{12},
$$

where the (real) coefficient of the $n$th power of $\cos \theta_{12}$ has been denoted by $c_{n}$.

Obviously, if $\Delta_{c d} \approx 0$ in the vicinity of $\theta_{12}=90^{\circ}$ then the coefficient $c_{0}$ must be small compared to $c_{1}$ and $c_{2}$. We find this to be the case. For example, for the case of an excess 
TABLE II. Predicted values of $\theta_{12}$ (in degrees) for the positions of the nongeometrical zeros of the CD parameter $\Delta_{c d}$ [cf. Eq. (41)] for various excess energies. Values obtained from the approximate Eqs. (43) and (44) (see text for discussions).

\begin{tabular}{lccccc}
\hline \hline $\begin{array}{l}E_{\text {exc }} \\
(\mathrm{eV})\end{array}$ & $\begin{array}{c}E_{1} \\
(\mathrm{eV})\end{array}$ & $\begin{array}{c}\theta_{12}^{(1)}, \\
\text { Eq. (43) }\end{array}$ & $\begin{array}{c}\theta_{12}^{(1)}, \\
\text { Eq. (44) }\end{array}$ & $\begin{array}{c}\theta_{12}^{(2)}, \\
\text { Eq. (43) }\end{array}$ & $\begin{array}{c}\theta_{12}^{(2)}, \\
\text { Eq. (44) }\end{array}$ \\
\hline 9 & 6 & 39.6 & 49.2 & 88.2 & 88.2 \\
& 8 & & & & \\
20 & 14.5 & 33.5 & 52.2 & 86.1 & 86.1 \\
& 17 & 35.0 & 51.6 & 86.2 & 86.2 \\
& 17.5 & 35.4 & 51.4 & 86.2 & 86.2 \\
40 & 30 & 19.3 & 55.3 & 83.9 & 83.8 \\
& 35 & 23.4 & 54.4 & 84.0 & 83.9 \\
50 & 45 & 15.8 & 55.2 & 83.3 & 83.2 \\
60 & 50 & & 55.4 & 81.8 & 81.6 \\
& 55 & & 55.8 & 82.7 & 82.6 \\
70 & 65 & & 56.2 & 82.3 & 82.2 \\
80 & 70 & & 57.5 & 82.0 & 81.8 \\
\hline \hline
\end{tabular}

energy of $20 \mathrm{eV}$, for $E_{1}=14.5 \mathrm{eV}$ and $E_{2}=5.5 \mathrm{eV}$, the coefficients $c_{n}$ are calculated to have the following values: $c_{0}$ $=-4.26, c_{1}=70.2, c_{2}=-103.2, c_{3}=33.3$, and $c_{4}=-4.28$, so that $\left|c_{0}\right| \ll\left|c_{1}\right|,\left|c_{2}\right|$. Similarly small values of the coefficient $c_{0}$ (compared to those of $c_{1}$ and $c_{2}$ ) are found for other energy sharings.

The exact positions $\theta_{12}$ of the dynamical zeros of $\Delta_{c d}$ for a given excess energy and energy sharing may be obtained by finding the real roots of the transcendental equation $\Delta_{c d}$ $=0$, where $\Delta_{c d}$ is given by Eq. (43). If one neglects the contributions of $f$ waves in the final state [i.e., one sets $a_{2}=b_{2}$ $=0$ in Eq. (27)], which gives a good approximation for an excess energy of $20 \mathrm{eV}$ [40], the equation determining the approximate positions of the zeros of the CD parameter becomes quadratic ( since $c_{3}=c_{4}=0$ ), which yields the solutions

$$
\theta_{12}^{(1,2)}=\arccos \left(\frac{-c_{1} \pm \sqrt{c_{1}^{2}-4 c_{0} c_{2}}}{2 c_{2}}\right),
$$

where $\quad c_{0}=\operatorname{Im}\left\{a_{0} b_{0}^{*}\right\}, \quad c_{1}=3 \operatorname{Im}\left\{a_{1} b_{0}^{*}+a_{0} b_{1}^{*}\right\}, \quad$ and $\quad c_{2}$ $=9 \operatorname{Im}\left\{a_{1} b_{1}^{*}\right\}$. The predicted positions of the nongeometrical zeros, obtained using the approximate Eqs. (43) and (44), are presented in Table II. Our results indicate that Eqs. (43) and (44) have two real roots for almost all excess energies up to $50 \mathrm{eV}$ (except for one kinematical situation for $E_{\text {exc }}=9 \mathrm{eV}$ ), while for higher energies there are several situations when there is only one real root. As predicted by Eq. (43), one of the two zeros usually appears in the interval of $14.6^{\circ}<\theta_{12}<40.1^{\circ}$, while the second zero appears in the interval of $81.3^{\circ}<\theta_{12}<88.4^{\circ}$ as the excess energy is varied from $9 \mathrm{eV}$ to $80 \mathrm{eV}$. One sees that the predictions for the CD parameter zero points near $\theta_{12}=85^{\circ}$, obtained using Eq. (44), are in agreement with those obtained using Eq. (43). On the contrary, the predictions for zeros near $\theta_{12}=40^{\circ}$, obtained using Eq. (44), are very different from (and less accurate than) those obtained

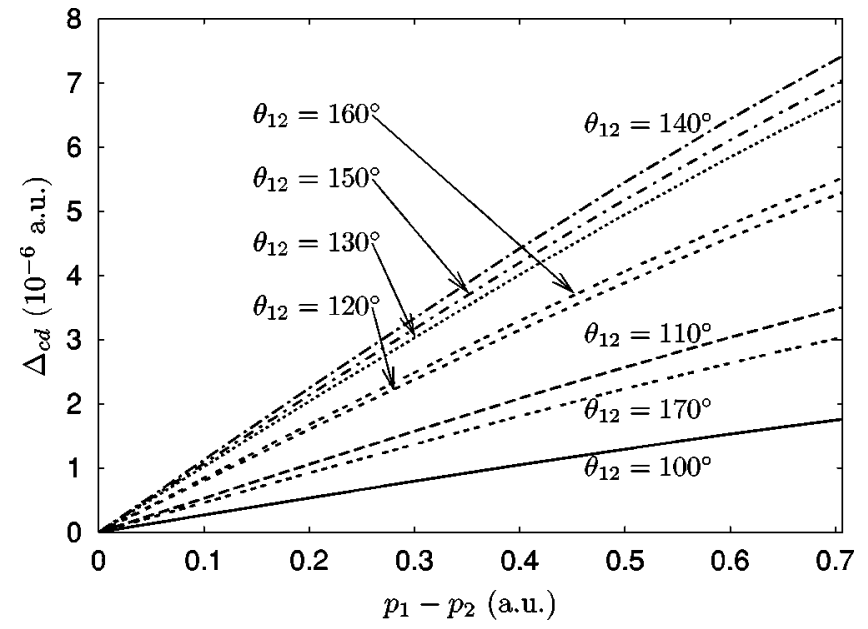

FIG. 14. Circular dichroism parameter $\Delta_{c d}$ for an excess energy of $20 \mathrm{eV}$ as a function of $p_{1}-p_{2}$ for various angles $\theta_{12}$.

with Eq. (43). This difference occurs because for small mutual ejection angles the contribution of the $d f$ channel [which is neglected in Eq. (44)] is important. As mentioned above, an independent calculation is necessary to confirm or reject the existence of the zeros in the CD parameters predicted by our LOPT approach.

\section{CD as a function of the energy sharing}

One can use the parametrization (27) for the polarizationinvariant amplitudes $f_{1}$ and $f_{2}$ to analyze the dependence of the CD effect upon the energy sharing between photoelectrons. In Fig. 14 we present the parameter $\Delta_{c d}$ as a function of the difference of the final-state electron momenta, $p_{1}-p_{2}$, for an excess energy of $20 \mathrm{eV}$ for various mutual ejection angles in the interval $100^{\circ} \leqslant \theta_{12} \leqslant 170^{\circ}$. The most interesting fact is that, as can be seen from Fig. 14, the parameter $\Delta_{c d}$ depends nearly linearly upon $p_{1}-p_{2}$ over a very wide range of energy sharing configurations, from $E_{1}=E_{2}$ $=10 \mathrm{eV}$ up to $E_{1}=17.0 \mathrm{eV}$ and $E_{2}=3 \mathrm{eV}$, for all mutual ejection angles considered.

\section{DISCUSSION}

In general, PDI is an essentially two-electron process whose description requires a proper account for electron correlations in both the initial ${ }^{1} S_{0}$ state and the $P$ wave, final two-electron continuum state. However, we have demonstrated that account of electron correlation only in the final state and only in the LOPT provides TDCSs which are in reasonable agreement with both the existing experimental data and the results of numerically intensive, ab initio calculations for excess energies up to $80 \mathrm{eV}$. We have interpreted this somewhat surprising and unexpected result as evidence that the dominant physical mechanism of PDI for the energy interval considered is the absorption of a photon by one of the electrons with subsequent, correlation-induced redistribution of the energy between the two electrons. Our results show that taking account of interelectron interaction only in the lowest PT order is sufficient to reproduce the experimen- 
tal data over a wide interval of kinematical conditions and excess energy sharings, with the only exception being the case of small mutual ejection angles, for which the TDCS is suppressed by Coulomb repulsion and for which a LOPT description is inadequate. This simple approximation is also capable of describing the delicate interference effects responsible for circular dichroism. Of course, it is not possible to present a priori theoretical arguments why high-order correlation effects are unimportant for the kinematical situations we have considered, owing to the fact that the correlation parameter, $1 / Z$, for $\mathrm{He}(Z=2)$ is not a small parameter. However, below we present our perspective on the treatment of electron-electron interactions using a LOPT approach and attempt to explain its success in the present work.

\section{A. On the applicability of the LOPT approach to treatment of interelectron interactions in PDI of $\mathrm{He}$}

When discussing the problem of convergence of PT expansions for Coulomb perturbations in atomic processes, one should distinguish between PT account of electron-nuclear, $V_{e n}$, and electron-electron, $V_{e e}$, interactions. Usually, the former approach is used in collisional problems, in which $V_{e n}$ interactions (or both $V_{e n}$ and $V_{e e}$ ) are taken into account using a plane-wave basis set (i.e., employing free-electron Green functions). The accuracy of such Born-like expansions is rather unclear and depends on the parameters of a particular problem; even the question whether such a PT series is convergent, asymptotic, or even initially divergent, is open. This circumstance may create an impression that PT treatment of Coulomb interactions is always questionable. However, since the convergence properties of any PT expansion essentially depend not only on the form of the perturbation operator, but also on the choice of the unperturbed basis set, the situation is quite different when $V_{e e}$ is taken into account using a Coulomb basis set (i.e., employing Coulomb Green functions). In particular, this is the case for the PT treatment of the correlation interaction $V_{e e}$ in bound state and photoionization (including PDI) problems for $\mathrm{He}$ and $\mathrm{He}$-like ions.

The PT expansion of the ground-state energy of a nonrelativistic He-like ion in powers of $1 / Z$,

$$
E_{(1 s)^{2}}=-Z^{2}+\sum_{n=1} E^{(n)}, \quad E^{(n)}=\epsilon^{(n)} / Z^{n-2},
$$

may be expected to be a convergent series even for $Z=2$ [58]. Thus, the question is only the convergence rate, which is defined by the $Z$-independent atomic parameters $\epsilon^{(n)}$. For ground and $(2 p)^{2}{ }^{3} P$ states, these parameters are obtained in Ref. [59] up to $n=20$. It is interesting that each of the few first coefficients $\epsilon^{(n)}$ in the expansion (45) decreases by about one order of magnitude with increasing $n$. As a result, even for neutral $\mathrm{He}(Z=2)$, the sum of the three leading corrections to $E_{(1 s)^{2}}^{(0)}=-Z^{2}=-108.84 \mathrm{eV}$ [i.e., $E^{(1)}$ $=(5 / 8) Z=34.02 \mathrm{eV}, \quad E^{(2)}=-4.29 \mathrm{eV}, \quad$ and $\left.\quad E^{(3)}=0.12 \mathrm{eV}\right]$ practically coincides with the experimental value $E_{(1 s)^{2}}^{\text {expt }}$ $\simeq-79.02 \mathrm{eV}$. (For the $(2 p)^{2}{ }^{3} P$-state, the convergence is similar [59].) Thus, the account of only the two lowest-order (i.e., $Z^{-1}$ and $Z^{-2}$ ) correlation corrections gives a value of the ground-state energy of He to within a few percent, which is quite acceptable for many applications in which high (spectroscopic) precision is not necessary or can be achieved by using spectroscopic experimental data.

For bound-continuum transitions, a global estimate of the importance of contributions from the next-order (in $V_{e e}$ ) corrections to the LOPT result (especially for small $Z, Z \geqslant 2$ ) cannot be obtained, since those contributions depend on the parameters (mostly, on the electron energies) of the particular problem considered. Specifically, the important difference between PT treatments of $V_{e e}$ for bound-bound and boundcontinuum transitions is that in the latter case, in each order of PT in $V_{e e}$, the nuclear charge $Z$ enters the PT result not only as a multiplicative factor [as in Eq. (45)] but also through the electron energy-dependent parameters (the socalled Coulomb factors of the form $\eta=Z / \sqrt{2 E}$ ), which characterize the intensity of electron-nuclear interaction $V_{e n}$ in the intermediate (virtual) and final (continuum) states of the escaping electron(s). Generally, as the Coulomb factors decrease in magnitude, so do the corresponding transition amplitudes. Thus, for different energy intervals, both the magnitudes of LOPT and high-order PT terms and the relative importance of sequential terms in the PT expansion of a physical amplitude in the parameter $1 / Z$ may be different in general. For multicharged ions, these deviations may have little consequence since the contribution of each next-order term in $V_{e e}$ is suppressed by the overall factor $1 / Z$. However, this factor is not a small parameter for $Z \gtrsim 2$; thus the accuracy of LOPT results in this case requires a special analysis for each particular problem.

Below we shall summarize some arguments concerning the accuracy of LOPT TDCS results for the PDI of He. Of course, the most convincing analysis would be the direct calculation of high-order corrections in $1 / Z$; however, this challenging problem is scarcely realizable at the present time since treatment of even the next-order (in $Z^{-1}$ ) correction requires an accurate estimation of multiple integrals involving three CGFs $G_{\mathcal{E}}\left(\mathbf{r}, \mathbf{r}^{\prime}\right)$, including integration over the energy parameters in two of them. Instead, we show that some conclusions may be made, based on the general remarks discussed above, on the existing PT analyses for angleintegrated cross sections and on comparisons of our LOPT TDCS results [40] with experimental and other $a b$ initio theoretical data. In the LOPT treatment of PDI, the Coulomb factors are $\eta_{1}=Z / p_{1}, \eta_{2}=Z / p_{2}$, and $\widetilde{\eta} \equiv-i Z \nu=-i Z / \sqrt{-2 \mathcal{E}}$, where $\mathcal{E}$ is the virtual electron energy (the energy parameter of the CGF $G_{\mathcal{E}}$ ). Effects of $V_{e n}$ are negligible only in the (Born) limit of small Coulomb factors. For the VUV region of photon energies explored in the present work, the Coulomb factors are of the order of unity or less; thus $V_{e n}$ should be taken into account exactly. Moreover, the interplay between Coulomb factors essentially determines the energy dependence and the magnitude of the dynamical atomic parameters (i.e., the radial matrix elements; see Appendix A) and, thus, the relative magnitudes of the contributions of the diagrams in Figs. 1(a) and 1(b) to the total PDI amplitude.

For the FSC [Fig. 1(a)] and GSC [Fig. 1(b)] diagrams, the values of the Coulomb factors corresponding to the intermediate states, $\widetilde{\eta}_{F}$ and $\tilde{\eta}_{G}$, are quite different, since $\tilde{\eta}_{G}$ is purely imaginary: $\tilde{\eta}_{G}=-i Z / \nu_{G}$, where $\nu_{G}=1 / \sqrt{-2 E_{G}}$ 
$=1 / \sqrt{-2\left(2 \epsilon_{1 s}-E_{p_{2}}\right)}$. The radial CGF, $g_{l}\left(\mathcal{E} ; r, r^{\prime}\right)$, with negative $\mathcal{E}=E_{G}$, is localized mostly at small $r$ and $r^{\prime}$. It thus has a much larger overlap with the $|1 s\rangle$ orbital as compared to the case of FSC, where $E_{F}$ (and thus $\tilde{\eta}_{F}$ ) is positive and $g_{l}\left(E_{F} ; r, r^{\prime}\right)$ oscillates as a function of $r$ and $r^{\prime}$. Quantitatively, this formal analysis shows that for moderate excess energies (for which the oscillations of the Coulomb wave functions of the final state are not too fast), the magnitude of the (real) GSC radial matrix elements is higher than that for FSC. Owing to the large magnitude of the LOPT result for GSC, it is clear that the LOPT treatment of GSC is inadequate, as the first-order contribution overestimates the extent of ground-state electron correlations, and higher-order terms must be considered [9]. For this reason we use a simple variational ground-state wave function that gives - empirically - a reasonable description of both the initial-state energy and its spatial distribution. The use of this initial-state together with inclusion of only first-order electron correlations in the final state appears empirically to be sufficiently accurate for current experimental measurements at intermediate excess energies. However, with increasing excess energy, the situation changes, and in order to achieve better agreement with experiments it is necessary to account for both FSC and GSC on an equal footing. This fact again may be understood by considering the behavior of the spatial integrals in the radial matrix elements involving the CGFs. Indeed, with increasing excess energy, the radial CGF $g_{l}\left(E_{G} ; r, r^{\prime}\right)$ becomes localized even closer to the nucleus; however, on the other hand, the integration over $r^{\prime}$ involves also the Coulomb continuum state, $R_{E_{f}, l_{f}}\left(r^{\prime}\right)$, whose oscillations for large $E_{f}$ significantly suppress the absolute value of the GSC amplitude. Since it is reasonable to expect that for higher-order (in 1/Z) GSC diagrams this tendency is even more significant than for the LOPT term, their contributions to the total GSC amplitude are thus not as important as for small excess energies. Consequently, at higher excess energy, the FSC and GSC LOPT contributions to the total amplitude of PDI become comparable and should strongly interfere. (Indeed, we have observed this in our numerical results for an excess energy of $450 \mathrm{eV}$.) We believe that these variations in LOPT treatments for intermediate and high energies and their formal analysis correspond physically to a change of the physical mechanism of PDI with increasing excess energy, i.e., to the growing role of the shakeoff mechanism at high energies.

Regarding final-state correlations, we note that the accuracy of our LOPT results is not uniform with respect to the mutual ejection angle $\theta \equiv \theta_{12}$. Indeed, final-state correlations are strongest when the two ionized electrons leave the atom along directions having a small mutual angle. This is precisely the electron configuration for which our LOPT results are not in good agreement with either experiment or with other, more detailed calculations. However, for intermediate excess energies and not extremely asymmetric energy sharings, the TDCS for this configuration is small, since the largest values of the TDCS (aside from requirements of symmetry selection rules) occur for configurations in which the electrons leave with large mutual angles. For large mutual ejection angles, the photoelectrons are well separated from each other, making the correlation operator truly a perturbative quantity. As for the angle-integrated cross sections, it may be expected that they should be described reasonably well by LOPT since the contribution of small mutual angles (for which TDCSs decrease) should be small. As a justification of this statement we can consider the good agreement of exact theoretical results [60] for the ratio of double-to-single photoionization cross sections, $\sigma^{++} / \sigma^{+}$, for $\mathrm{He}, \mathrm{Li}^{+}$, and $\mathrm{O}^{6+}$ with those obtained from the parametrization of exact results based on the scaling law in Eq. (15). This scaling law follows immediately from the LOPT analysis and was suggested in Ref. [47] assuming that $Z \rightarrow \infty$ (which is equivalent to taking account of $V_{e e}$ only in the lowest nonvanishing order, i.e., to the LOPT result). In Ref. [47] it was also mentioned that there is reasonable agreement of numerical and scaled results for TDCSs. Finally, in a very recent publication [61] it has been shown that straightforward LOPT calculations of the ratio $\sigma^{++} / \sigma^{+}$are in reasonable agreement with experimental data for $\mathrm{He}$ for excess energies from threshold up to $300 \mathrm{eV}$.

\section{B. On the physical mechanisms of PDI of He}

Since in LOPT the PDI amplitude for intermediate excess energies is described by two diagrams [i.e., that in Fig. 1(a) and that with interchanged momenta $\mathbf{p}_{1}$ and $\left.\mathbf{p}_{2}\right]$, both of which describe the so-called direct (on-shell) and virtual (off-shell) knock-out mechanisms, analysis of their relative contributions allows one to elucidate the underlying physical mechanism of PDI. The analogy between the direct knockout mechanism for PDI and the similar mechanism for the $(e, 2 e)$ process has been noted some time ago [62]. However, in the case of PDI this analogy is incomplete, since we have a quite different kinematical situation: the direction of an intermediate electron momentum is not fixed and requires an integration over the differential solid angle. As discussed above, our approach involves the on-shell knock-out mechanism: taking into account only the imaginary part of the CGF in Eq. (7), the amplitude $M_{F}$ may be expressed as

$$
M_{F}^{a b}=\int d \Omega_{\mathbf{k}^{\prime}} M_{\epsilon_{1 s} \rightarrow \mathbf{k}^{\prime}} M_{\mathbf{k}^{\prime} \rightarrow\left(\mathbf{p}_{1}, \mathbf{p}_{2}\right)},
$$

where the integrand is a product of the single-electron photoionization amplitude $\left(M_{\epsilon_{1 s} \rightarrow \mathbf{k}^{\prime}}\right)$ and an $(e, 2 e)$ amplitude $\left(M_{\mathbf{k}^{\prime} \rightarrow\left(\mathbf{p}_{1}, \mathbf{p}_{2}\right)}\right)$ and where the integration is over all directions of the intermediate electron momentum $\mathbf{k}^{\prime}$. However, we find that this on-shell knock-out process does not give the dominant contribution to the PDI amplitude. On the contrary, the off-shell (or virtual) knock-out mechanism is more important. This mechanism of PDI does not assume a real collision between two electrons; moreover, the continuum state of an electron with energy $\mathcal{E}=\epsilon_{1 s}+\omega$ does not contribute to the real part of the CFG, $G_{\mathcal{E}}$, even as an intermediate (virtual) state.

Exhaustive information on the dynamics of PDI may be obtained by analyzing the energy dependence of the radial matrix elements $\mathcal{R}_{F}^{\left(l_{1} l_{2}\right)}\left(p_{i}, p_{j}\right)$, which are independent of the particular geometry and give us complete information on the dynamical parameters. The important questions to be an- 
TABLE III. Radial matrix elements $\mathcal{R}_{F}^{\left(l_{1} l_{2}\right)}$ [cf. Eq. (A2)] for an excess energies of 20 and $80 \mathrm{eV}$. The theoretical model is the same as for Table I.

\begin{tabular}{cccccccc}
\hline \hline $\begin{array}{c}E_{\text {exc }} \\
(\mathrm{eV})\end{array}$ & $\begin{array}{c}E_{1} \\
(\mathrm{eV})\end{array}$ & $\begin{array}{c}\mathcal{R}_{F}^{(10)} \\
\left(10^{-3} \text { a.u. }\right)\end{array}$ & $\begin{array}{c}\mathcal{R}_{F}^{(01)} \\
\left(10^{-3} \text { a.u. }\right)\end{array}$ & $\begin{array}{c}\mathcal{R}_{F}^{(21)} \\
\left(10^{-3} \text { a.u. }\right)\end{array}$ & $\begin{array}{c}\mathcal{R}_{F}^{(12)} \\
\left(10^{-3} \text { a.u. }\right)\end{array}$ & $\begin{array}{c}\mathcal{R}_{F}^{(32)} \\
\left(10^{-3} \text { a.u. }\right)\end{array}$ & $\begin{array}{c}\mathcal{R}_{F}^{(23)} \\
\left(10^{-3} \text { a.u. }\right)\end{array}$ \\
\hline 20 & 17.5 & $0.81+2.95 i$ & $12.3+6.61 i$ & $-13.4-1.55 i$ & $-9.46+2.34 i$ & $-0.82-1.52 i$ & $-0.65-1.31 i$ \\
& 14.5 & $0.98+3.32 i$ & $12.9+7.66 i$ & $-14.3-1.42 i$ & $-11.6+2.54 i$ & $-1.01-1.64 i$ & $-0.91-1.69 i$ \\
& 10.0 & $1.13+3.33 i$ & $11.7+8.08 i$ & $-13.2-1.01 i$ & $-13.3+2.35 i$ & $-1.06-1.47 i$ & $-1.16-1.93 i$ \\
& 5.5 & $1.21+2.97 i$ & $9.38+7.61 i$ & $-10.7-0.60 i$ & $-13.3+1.88 i$ & $-0.87-1.10 i$ & $-1.20-1.83 i$ \\
& 2.5 & $1.13+2.46 i$ & $7.16+6.54 i$ & $-8.23-0.35 i$ & $-11.8+1.42 i$ & $-0.63-0.76 i$ & $-1.06-1.53 i$ \\
& 70 & $-0.68+2.79 i$ & $12.0+5.85 i$ & $-16.3-1.29 i$ & $-7.75+3.72 i$ & $-2.08-0.30 i$ & $-1.35-2.09 i$ \\
& 58 & $-0.63+2.91 i$ & $10.5+6.62 i$ & $-15.9-0.61 i$ & $-10.2+3.67 i$ & $-3.05-0.30 i$ & $-2.18-2.83 i$ \\
& 40 & $-0.10+2.58 i$ & $6.85+6.79 i$ & $-12.7+0.09 i$ & $-12.7+2.84 i$ & $-3.09-0.23 i$ & $-3.09-3.05 i$ \\
& 22 & $0.52+2.04 i$ & $3.06+6.29 i$ & $-8.41+0.42 i$ & $-13.4+1.81 i$ & $-2.01-0.14 i$ & $-3.17-2.59 i$ \\
& 10 & $0.78+1.54 i$ & $9.25+5.38 i$ & $-5.27+0.43 i$ & $-12.1+1.10 i$ & $-1.06-0.07 i$ & $-2.47-1.91 i$ \\
\hline \hline
\end{tabular}

swered are: (i) which individual electron angular momenta give the dominant contribution to the transition amplitude, (ii) what are the relative contributions of the real and imaginary parts of the CGF in the corresponding matrix elements, and (iii) how do the answers to the first two questions above depend upon the excess energy and the energy sharing? In Table III we present the numerical values of the radial matrix elements $\mathcal{R}_{F}^{\left(l_{1} l_{2}\right)}$ for excess energies of $20 \mathrm{eV}$ and $80 \mathrm{eV}$, for several of the lowest angular momentum pairs and for various energy sharings (the energy sharing ratios $R \equiv E_{1} / E_{2}$ for the case of $80 \mathrm{eV}$ are the same as for $20 \mathrm{eV}$ ). As may be seen from Table III, the matrix elements having angular momenta $\left(l_{1}, l_{2}\right)$ equal to $(1,0),(0,1),(2,1)$, and $(1,2)$ give the dominant contributions to the transition amplitude. The real parts of $\mathcal{R}_{F}^{(21)}$ and $\mathcal{R}_{F}^{(12)}$ are larger than their imaginary parts by approximately one order of magnitude, and the real parts of $\mathcal{R}_{F}^{(01)}$ are also larger then their imaginary parts. Only in the matrix elements $\mathcal{R}_{F}^{(10)}$ (which give the smallest contribution to the transition amplitude within this group) are the imaginary parts larger then the real parts. This fact implies that the on-shell knock-out process is relatively more important for the $\mathcal{R}_{F}^{(10)}$ matrix element than for the others, for which the off-shell knock-out mechanism dominates. Also, the probability of the latter PDI events is greater for those kinematical situations in which, after the inelastic electron-electron collision, the primary electron leaves with higher energy $\left(E_{1}\right)$ than the secondary electron, in agreement with classical arguments.

At higher excess energies, such as at $450 \mathrm{eV}$, account of correlation in both initial and final states becomes necessary and the LOPT approach provides reasonable agreement with experiment only for large mutual ejection angles. It is reasonable to expect that with increasing excess energy, the accuracy of a LOPT account for FSC should become even better than for intermediate energies. Thus, in order to extend our results to a wider interval of excess energies, a more exact account of GSC is necessary. Assuming that only the diagram in Fig. 1(a) is sufficient to account for FSC contributions, one may neglect FSC effects in calculations of highorder correlation corrections to the GSC diagram Fig. 1(b) or, equivalently, to replace the diagram Fig. 1(b) by an effective matrix element involving an uncorrelated final state and an appropriately highly correlated initial state, which may be used to describe the shake-off mechanism of PDI. In this way the PDI amplitude takes the form of a sum of the knockout and shake-off amplitudes. By neglecting their interference, we would recover the results of Ref. [63], in which the PDI cross section is approximated by a sum of independent shake-off and knock-out contributions (with the latter estimated classically). We note that calculation of the knock-out contribution in our approach, which involves partial-wave expansions, is not more complicated than the quasiclassical analysis of Ref. [63]. Furthermore, it gives reasonable results for the angle-resolved absolute TDCS, for which the accuracy of quasiclassical simulations is unclear.

\section{On the importance of the large- $r$ and small-r electron correlations}

In order to estimate the importance of large- $r$ and small- $r$ correlations for the PDI amplitude, we have compared the radial matrix elements $\mathcal{R}_{F, G}^{\left(l_{1}, l_{2}\right)}$ evaluated using the asymptotic $(r \rightarrow 0$ and $r \rightarrow \infty)$ expressions for the function $u_{p l}(r)$ [using Eqs. (A30) and (A31)] with the exact results. We have found that for intermediate excess energies, such as $20 \mathrm{eV}$, the large- $r$ correlation and small- $r$ correlation terms in the matrix elements $\mathcal{R}_{F}$ are of the same order of magnitude and that there is a strong interference between them, which implies the importance of both small- $r$ and large- $r$ correlations. On the contrary, for the case of higher excess energy, such as $450 \mathrm{eV}$, we find that the large- $r$ correlation terms are negligible in both $\mathcal{R}_{F}$ and $\mathcal{R}_{G}$ matrix elements, which implies the importance of the small- $r$ and intermediate- $r$ correlations.

\section{CONCLUSIONS}

To summarize, we have used a LOPT approach (in the interelectron interaction) to evaluate the TDCS for PDI of He over a wide range of excess energies and, for the case of circularly polarized photons, to analyze the circular dichroism effect. We have found that for an excess energy of the 
order of tens of $\mathrm{eV}$, the PDI process is dominated by the virtual (off-shell) knock-out mechanism, while the direct (on-shell) knock-out mechanism is rather small for the large mutual angles at which the $\mathrm{CD}$ effect is maximum. As a result of these findings, we can deduce that the $\mathrm{CD}$ effect in PDI at intermediate energies originates from the nonzero electron Coulomb phase shifts, i.e., from the interference of two two-electron, phase-locked wave packets: one composed of plane waves and the other that is elastically scattered from the nucleus.

\section{ACKNOWLEDGMENTS}

We wish to thank M. Achler, L. Avaldi, R. Dörner, and T. Reddish for providing us with their experimental data, and $\mathrm{J}$. Colgan and A. Kheifets for sending us the results of their theoretical calculations. This work was supported in part by the U.S. Department of Energy, Office of Science, Division of Chemical Sciences, Geosciences, and Biosciences, under Grant No. DE-FG03-96ER14646 and by the joint Grant No. VZ-010-0 of the CRDF and the RF Ministry of Education (N.L.M.). This work was completed utilizing the Research Computing Facility of the University of Nebraska—Lincoln.

\section{APPENDIX A: EVALUATION OF PARTIAL-WAVE RADIAL MATRIX ELEMENTS}

In this appendix we present the derivation and final results for the dynamical parameters $D_{F, G}^{\left(l_{1} l_{2}\right)}\left(p_{1}, p_{2}\right)$, introduced in Eq. (23), for arbitrary individual electron angular momenta $l_{1}$ and $l_{2}$.

\section{Evaluation of the FSC parameters}

Let us consider first the evaluation of the FSC parameters $D_{F}^{\left(l_{1} l_{2}\right)}$. The explicit form of $D_{F}^{\left(l_{1} l_{2}\right)}\left(p_{1}, p_{2}\right)$ follows from Eq. (7) after substituting the multipole expansions in Eqs. (16)-(18) into Eqs. (7) and (9), and using the fact that $\left(\mathbf{e} \cdot \nabla_{\mathbf{r}}\right) \varphi_{0}(r)=-Z_{s}(\mathbf{e} \cdot \hat{\mathbf{r}}) \varphi_{0}(r)$. After performing the angular integrations by means of Eq. (22), the expression for $D_{F}^{\left(l_{1} l_{2}\right)}$ may be written as follows:

$$
\begin{aligned}
D_{F}^{\left(l_{1} l_{2}\right)}\left(p_{1}, p_{2}\right)= & i^{l_{1}+l_{2}+1} \frac{\sqrt{2}}{(2 \pi)^{3}} \frac{4 \pi Z_{s}^{4}}{p_{1} p_{2}} e^{i\left(\delta_{l_{1}}\left(p_{1}\right)+\delta_{l_{2}}\left(p_{2}\right)\right)} \\
& \times \sqrt{\frac{2 l_{1}+1}{3\left(2 l_{2}+1\right) l_{>}}} C_{l_{1} 0 l_{2} 0}^{10} \mathcal{R}_{F}^{\left(l_{1} l_{2}\right)}\left(p_{1}, p_{2}\right),
\end{aligned}
$$

where $l_{2}=l_{1} \pm 1, l_{>}=\max \left(l_{1}, l_{2}\right)$ and $\mathcal{R}_{F}^{\left(l_{1} l_{2}\right)}$ is the radial integral,

$$
\begin{aligned}
\mathcal{R}_{F}^{\left(l_{1} l_{2}\right)}\left(p_{1}, p_{2}\right)= & \int_{0}^{\infty} d r r^{2} R_{p_{1} l_{1}}(r) u_{p_{2} l_{2}}(r) \\
& \times \int_{0}^{\infty} d r^{\prime} r^{\prime 2} g_{1}\left(E ; r, r^{\prime}\right) e^{-Z_{s} r^{\prime}} .
\end{aligned}
$$

In this equation, the function $u_{p_{2} l_{2}}(r)$ is related to the correlation potential $U_{\mathbf{p}_{2}}(\mathbf{r})$ as follows:

$$
U_{\mathbf{p}_{2}}(\mathbf{r})=2 \pi^{1 / 2} \frac{Z_{s}^{3 / 2}}{p_{2}} \sum_{l_{2}=0}^{\infty}(-i)^{l_{2}} e^{i \delta_{l_{2}}\left(p_{2}\right)} u_{p_{2} l_{2}}(r) P_{l_{2}}\left(\hat{\mathbf{p}}_{2} \cdot \hat{\mathbf{r}}\right) .
$$

The expression for $u_{p_{2} l_{2}}(r)$ thus follows from Eqs. (9) and (18),

$$
u_{p_{2} l_{2}}(r)=\int_{0}^{\infty} d r^{\prime \prime} r^{\prime \prime 2} R_{p_{2} l_{2}}\left(r^{\prime \prime}\right) \frac{r_{<}^{l_{2}}}{r_{>}^{l_{2}+1}} e^{-Z_{s} r^{\prime \prime}},
$$

where $r_{<}=\min \left(r, r^{\prime \prime}\right)$ and $r_{>}=\max \left(r, r^{\prime \prime}\right)$. Thus, the radial matrix elements in Eq. (A2) involve three radial integrations. In order to extract an explicit dependence of $u_{p_{2} l_{2}}(r)$ on $r$, we use the standard integral representation for the confluent hypergeometric function, $\Phi$, that enters the expression (19) for $R_{p_{2} l_{2}}(r)$ to obtain

$$
R_{p l}(r)=\frac{(2 p)^{l+1} e^{\pi \eta / 2} r^{l} e^{-i p r}}{|\Gamma(l+1+i \eta)|} \int_{0}^{1} d x e^{2 i p r x} x^{l+i \eta}(1-x)^{l-i \eta} .
$$

The integration over $r^{\prime \prime}$ in Eq. (A4) can be performed analytically in terms of elementary functions [see Eqs. (3.351.1-2) in Ref. [64]]; we thus obtain the following expression for $u_{p l}(r)$,

$$
u_{p l}(r)=\mathcal{C}_{p l} \int_{0}^{1} d x x^{l+i \eta}(1-x)^{l-i \eta} \chi_{p l}(r, x)
$$

where $\mathcal{C}_{p l}$ and $\chi_{p l}(r, x)$ are given by

$$
\mathcal{C}_{p l}=\frac{(2 p)^{l+1} e^{\pi \eta / 2}}{|\Gamma(l+1+i \eta)|}
$$

$$
\begin{aligned}
\chi_{p l}(r, x)= & \int_{0}^{\infty} d r^{\prime \prime} r^{\prime \prime l+2} e^{-\tau r^{\prime \prime}} \frac{r_{<}^{l}}{r_{>}^{l+1}}=\frac{(2 l+2) !}{\tau\left(\tau^{2} r\right)^{l+1}} \\
& \times\left\{1-e^{-\tau r} \sum_{k=0}^{2 l+1}\left(1-\frac{\delta_{k, 2 l+1}}{2 l+2}\right) \frac{(\tau r)^{k}}{k !}\right\},
\end{aligned}
$$

and where $\tau=Z_{s}+i p(1-2 x)$. Using Eq. (A6) and the integral representation (20) for the radial part of the CGF, $g_{1}\left(E ; r, r^{\prime}\right)$, the integrations over the variables $r^{\prime}$ and $r$ in Eq. (A2) may be performed analytically in terms of elementary functions, leaving for numerical calculation only the integrations over $x$ [see Eq. (A6)] and the variable $u$ in the integral representation (20) for $g_{l}\left(E ; r, r^{\prime}\right)$.

The integrals involving the modified Bessel function $I_{2 l+1}$ [cf. Eq. (20)] are evaluated using the formula [64] 


$$
\begin{aligned}
& \int_{0}^{\infty} d t t^{n-1 / 2} e^{-\sigma t} I_{2 l+1}(2 \beta \sqrt{t}) \\
& \quad=\frac{(n+l) !}{(2 l+1) !} \frac{\beta^{2 l+1}}{\sigma^{n+l+1}} e^{\beta^{2} / \sigma} \Phi\left(l+1-n ; 2 l+2 ;-\frac{\beta^{2}}{\sigma}\right),
\end{aligned}
$$

where the confluent hypergeometric function $\Phi(l+1-n ; 2 l$ $+2 ; z)$ with integer parameters is either a polynomial in $z$ [for $n \geqslant(l+1)]$ or reduces to elementary functions. Such reduction may be performed by using the recurrence relations for $\Phi$ to express it in terms of $\Phi(1 / 2+\nu ; 1+2 \nu ; z)$ with halfinteger $\nu$, which may be expressed as a Bessel function of half-integer order $\nu$ (see Eq. (10) on p. 265 in Ref. [65]), which in turn is a combination of polynomials in $z$ and exponentials.

The integration over $r^{\prime}$ in Eq. (A2) [with the substitution of Eq. (20) and the use of Eq. (A9)] is straightforward,

$$
\begin{aligned}
\int_{0}^{\infty} d r^{\prime} r^{\prime 2} g_{1}\left(E ; r, r^{\prime}\right) e^{-Z_{s} r^{\prime}} \\
\quad=\frac{16 \nu r}{\left(1+Z_{s} \nu\right)^{4}} \int_{0}^{1} d u \frac{u^{1-Z \nu}}{(1+\gamma u)^{4}} \exp \left(-\frac{r}{\nu} \frac{1-\gamma u}{1+\gamma u}\right),
\end{aligned}
$$

where

$$
\gamma=\left(1-Z_{s} \nu\right) /\left(1+Z_{s} \nu\right),
$$

and where $\nu \equiv \nu_{F}=1 / \sqrt{-2\left(E_{p_{1}}+E_{p_{2}}-\epsilon_{1 s}\right)}=i\left|\nu_{F}\right|$. Thus, the parameter $\nu$ for the FSC diagram in Fig. 1(a) is imaginary, which leads to the complexity of $\mathcal{R}_{F}^{\left(l_{1} l_{2}\right)}\left(p_{1}, p_{2}\right)$, whose imaginary part corresponds to the direct (on-shell) knockout PDI amplitude.

After substituting Eqs. (19) and (A10) into Eq. (A2) and taking into account Eqs. (A6)-(A8), the integration over $r$ which involves $R_{p_{1} l_{1}}(r)$ may be performed using the formula [64]

$$
J_{k m}^{\kappa \beta}(\sigma)=\int_{0}^{\infty} d t t^{k-1} e^{-\sigma t} \Phi(\kappa ; m ; \beta t)=\frac{(k-1) !}{\sigma^{k}}{ }_{2} F_{1}\left(\kappa, k ; m ; \frac{\beta}{\sigma}\right),
$$

where the hypergeometric function ${ }_{2} F_{1}$ reduces to elementary functions for any positive integer values of $k$ and $m$ (as in our case). For $m \leqslant k$, it reduces to a hypergeometric polynomial of the order of $(k-m)$ by using the known Gauss relation ${ }_{2} F_{1}(\kappa, k ; m ; z)=(1-z)^{m-k-\kappa}{ }_{2} F_{1}(-(k-m), m-\kappa ; m ; z)$. For $m>k$, the reduction is obtained by using the analytical continuation formula (cf. Eq. 2.1.4(17) in Ref. [65])

$$
\begin{aligned}
& \frac{1}{(m-1) !}{ }_{2} F_{1}(\kappa, k ; m ; z) \\
&= \frac{\Gamma(k-\kappa)(1-z)^{m-k-\kappa}}{(k-1) ! \Gamma(m-\kappa)(-z)^{m-k}} \\
& \quad \times{ }_{2} F_{1}(-k+1, m-k ; 1-k+\kappa ; 1 / z) \\
&+\frac{\Gamma(\kappa-k){ }_{2} F_{1}(k+1-m, k ; 1+k-\kappa ; 1 / z)}{(m-k-1) ! \Gamma(\kappa)(-z)^{k}} .
\end{aligned}
$$

The resulting two-dimensional integral for $\mathcal{R}_{F}^{\left(l_{1} l_{2}\right)}$ may be written in the following form:

$$
\begin{aligned}
\mathcal{R}_{F}^{\left(l_{1} l_{2}\right)=} & \frac{16 \nu C_{p_{1} l_{1}}\left(2 p_{1}\right)^{l_{1}} C_{p_{2} l_{2}}}{\left(1+Z_{s} \nu\right)^{4}} \frac{\left(2 l_{2}+2\right) !}{\left(2 l_{1}+1\right) !} \int_{0}^{1} d u \frac{u^{1-Z \nu}}{(1+\gamma u)^{4}} \\
& \times \int_{0}^{1} d x \frac{x^{l_{2}+i \eta_{2}}(1-x)^{l_{2}-i \eta_{2}}}{\tau^{2 l_{2}+3}}\left\{J_{l_{1}-l_{2}+3,2 l_{1}+2}^{l_{1}+1+i \eta_{1}, 2 i p_{1}}(\lambda)\right. \\
& \left.-\sum_{k=0}^{2 l_{2}+1}\left(1-\frac{\delta_{k, 2 l_{2}+1}}{2 l_{2}+2}\right) \frac{\tau^{k}}{k !} J_{l_{1}-l_{2}+k+3,2 l_{1}+2}^{l_{1}+1+i \eta_{1}, 2 i p_{1}}(\lambda+\tau)\right\},
\end{aligned}
$$

where

$$
\lambda=i p_{1}+\frac{1}{\nu} \frac{1-\gamma u}{1+\gamma u} .
$$

Note that the functions $J_{k, m}^{\kappa, \beta}(\sigma)$ defined in Eq. (A12) may be reduced to elementary functions as discussed below Eq. (A12).

\section{Evaluation of the GSC parameters}

In order to evaluate the GSC amplitude in Eq. (8), we first carry out the angular integrations in Eq. (8) by means of the Wigner-Eckart theorem. Next, we integrate over $r$ by parts to move the action of the differential operator $d / d r$ from the CGF to the function $R_{p_{1} l_{1}}(r)$ (i.e., the radial part of $\psi_{\mathbf{p}_{1}}^{(-)}$). Thus, the function $D_{G}^{\left(l_{1} l_{2}\right)}\left(p_{1}, p_{2}\right)$, introduced in Eqs. (21) and (23), has the following form:

$$
\begin{aligned}
D_{G}^{\left(l_{1} l_{2}\right)}\left(p_{1}, p_{2}\right)= & i^{l_{1}-l_{2}+1} \frac{\sqrt{2}}{(2 \pi)^{3}} \frac{4 \pi Z_{s}^{3}}{p_{1} p_{2}\left(2 l_{2}+1\right)} \\
& \times e^{i\left(\delta_{l_{1}}\left(p_{1}\right)+\delta_{l_{2}}\left(p_{2}\right)\right)} \mathcal{R}_{G}^{\left(l_{1} l_{2}\right)}\left(p_{1}, p_{2}\right),
\end{aligned}
$$

where the radial integral $\mathcal{R}_{G}^{\left(l_{1} l_{2}\right)}$ is defined as

$$
\begin{aligned}
\mathcal{R}_{G}^{\left(l_{1} l_{2}\right)}\left(p_{1}, p_{2}\right)= & \int_{0}^{\infty} d r r^{2} \hat{D}\left(l_{2}, l_{1}\right) R_{p_{1} l_{1}}(r) \\
& \times \int_{0}^{\infty} d r^{\prime} r^{\prime 2} g_{l_{2}}\left(E ; r, r^{\prime}\right) u_{p_{2} l_{2}}\left(r^{\prime}\right) e^{-Z_{s} r^{\prime}},
\end{aligned}
$$

the radial momentum operator $\hat{D}\left(l_{2}, l_{1}\right)$ is given by 


$$
\hat{D}\left(l_{2}, l_{1}\right)=\frac{d}{d r}+\frac{\operatorname{sgn}\left(l_{1}-l_{2}\right) l_{>}+1}{r},
$$

and $\operatorname{sgn}(z)$ is the sign of $z$. The integral over $r^{\prime}$ in Eq. (A17) involves $u_{p l}\left(r^{\prime}\right)$ and is thus much more complicated than the corresponding FSC integral over $r^{\prime}$ in Eq. (A10). Substituting Eqs. (20) and (A6) into Eq. (A17) and using Eq. (A9) gives the following result for the integral over $r^{\prime}$ in Eq. (A17),

$$
\begin{aligned}
& \int_{0}^{\infty} d r^{\prime} r^{\prime 2} g_{l_{2}}\left(E ; r, r^{\prime}\right) u_{p_{2} l_{2}}\left(r^{\prime}\right) e^{-Z_{s} r^{\prime}} \\
&=8 \nu \mathcal{C}_{p_{2} l_{2}}\left(l_{2}+1\right) \\
& \times(4 r)^{l_{2}} \int_{0}^{1} d u u^{l_{2}-Z \nu} \int_{0}^{1} d x \frac{x^{l_{2}+i \eta_{2}}(1-x)^{l_{2}-i \eta_{2}}}{\tau^{2 l_{2}+3}} \\
& \times\left[\exp \left(-\frac{r}{\nu} \frac{1-\gamma u}{1+\gamma u}\right) \frac{\Phi\left(2 l_{2} ; 2 l_{2}+2 ;-\frac{r}{\nu} \frac{\beta_{1}}{\nu 1-u}\right)}{\alpha^{2}[\nu(1-u)]^{2 l_{2}}}\right. \\
&-\exp \left(-\frac{r}{\nu} \frac{1-\gamma u+\xi \tau(1+u)}{1+\gamma u+\xi \tau(1-u)}\right) \\
&\left.\times \sum_{k=-1}^{2 l_{2}} b_{k}^{\left(l_{2}\right)} \frac{\Phi\left(k ; 2 l_{2}+2 ;-\frac{r}{\nu} \frac{\beta_{2}}{1-u}\right)}{[\nu(1-u)]^{k}}\right],
\end{aligned}
$$

where

$$
\begin{gathered}
\alpha=\left(1+Z_{s} \nu\right)(1+\gamma u), \quad \xi=\frac{\nu}{1+Z_{s} \nu}, \\
\beta_{1}=\frac{4 u}{\left(1+Z_{s} \nu\right)(1+\gamma u)}, \\
\beta_{2}=\frac{4 u}{\left(1+Z_{s} \nu\right)[1+\gamma u+\xi \tau(1-u)]}, \\
b_{k}^{\left(l_{2}\right)}=\left(1-\frac{\delta_{k,-1}}{2 l_{2}+2}\right) \frac{\left(2 l_{2}+1-k\right) \tau^{2 l_{2}-k}}{[\alpha+\nu \tau(1-u)]^{2 l_{2}+2-k}},
\end{gathered}
$$

and $\gamma$ is defined by Eq. (A11). Note that for the GSC amplitude given in Eq. (8) the parameter $\nu$ appearing in the integral representation for the radial part of the CGF [cf. Eq. (20)] is a positive real number, $\nu \equiv \nu_{G}=1 / \sqrt{-2\left(2 \epsilon_{1 s}-E_{p_{2}}\right)}$. Thus the radial matrix elements $\mathcal{R}_{G}^{\left(l_{1} l_{2}\right)}\left(p_{1}, p_{2}\right)$ are real despite the appearance of complex quantities in their definitions [see Eqs. (A22), (A25), and (A28) below].

In order to perform an analytical integration over $r$ in Eq. (A17), it is convenient to employ the special relations for the action of the operator $\hat{D}\left(l_{2}, l_{1}\right)$ on $\mathcal{R}_{p_{1} l_{1}}(r)$ in Eq. (A17) [see Eqs. (A1) and (A2) in Ref. [62]]. Specifically, for $l_{1}=l_{2}+1$ (i.e., $l_{2}=l, l_{1}=l+1$ ),

$$
\begin{aligned}
D(l, l+1) R_{p_{1} l+1}(r)= & p_{1} \frac{\left|c_{1}\right|}{c_{1}^{*}} R_{p_{1} l}(r)-\frac{2 Z C_{p_{1} l+1}}{(2 l+1) ! c_{1}^{*}}\left(2 p_{1} r\right)^{l-1} e^{-i p_{1} r} \\
& \times\left[\Phi\left(c_{2} ; 2 l+2 ; 2 i p_{1} r\right)\right. \\
& \left.-\Phi\left(c_{1}, 2 l+2 ; 2 i p_{1} r\right)\right] .
\end{aligned}
$$

In Eq. (A21) and below we use the abbreviation, $c_{k} \equiv l+k$ $+i \eta_{1}$. We now consider the first term on the right-hand side of Eq. (A21) separately from the remaining two terms. We shall thus represent $\mathcal{R}_{G}^{\left(l_{1}, l_{2}\right)}$ as the sum of two contributions, each of whose evaluations we consider below.

Since the first term on the right-hand side of Eq. (A21) is proportional to $R_{p_{1} l}(r)$, the evaluation of its contribution to the integral (A17) is simplified by taking into account the known relation $\left\langle R_{p l}(r)\right| g_{l}\left(\mathcal{E} ; r, r^{\prime}\right)=\left(E_{p}-\mathcal{E}\right)^{-1}\left\langle R_{p l}\left(r^{\prime}\right)\right|$. The corresponding contribution of this term to $\mathcal{R}_{G}^{(l+1, l)}$ is thus given by $p_{1}\left(\left|c_{1}\right| / c_{1}^{*}\right) \mathcal{S}_{G}^{(l)}$, where we have defined the matrix element $\quad \mathcal{S}_{G}^{(l)} \equiv\left\langle R_{p_{1} l}\left(r^{\prime}\right)\left|u_{p_{2} l}\left(r^{\prime}\right)\right| \varphi_{0}\left(r^{\prime}\right)\right\rangle /\left(E_{p_{1}}+E_{p_{2}}-2 \epsilon_{1 s}\right)$, which reduces to a one-dimensional integral (over $x$ ) by using Eqs. (A6), (A8), and (A12),

$$
\begin{aligned}
\mathcal{S}_{G}^{(l)}= & \frac{C_{p_{1}} \mathcal{C}_{p_{2} l}\left(2 p_{1}\right)^{l}(2 l+2)}{\left(E_{p_{1}}+E_{p_{2}}-2 \epsilon_{1 s}\right)} \\
& \times \int_{0}^{1} d x \frac{x^{l+i \eta_{2}}(1-x)^{l-i \eta_{2}}}{\tau^{2 l+3}}\left[J_{2,2 l+2}^{c_{1}, 2 i p_{1}}\left(\lambda_{0}\right)\right. \\
& \left.-\sum_{k=0}^{2 l+1}\left(1-\frac{\delta_{k, 2 l+1}}{2 l+2}\right) \frac{\tau^{k}}{k !} J_{k+2,2 l+2}^{c_{1}, 2 i p_{1}}\left(\lambda_{0}+\tau\right)\right],
\end{aligned}
$$

where $\lambda_{0}=i p_{1}+Z_{s}$.

Since the confluent hypergeometric functions $\Phi$ in Eqs. (A21) and (A19) have the same lower parameter, $m=2 l+2$, integrals involving the product of two functions $\Phi$ are calculated using a known result (see Eq. 6.15(22) in Ref. [61]). For our purposes, we present this result as

$$
\begin{aligned}
\int_{0}^{\infty} & d r e^{-\sigma r} r^{m-1} \Phi\left(\kappa ; m ; 2 i p_{1} r\right) \Phi\left(k ; m ;-\frac{\beta}{\nu(1-u)} r\right) \\
= & {[\nu(1-u)]^{k} K_{k \beta}^{\kappa m}(\sigma), }
\end{aligned}
$$

where

$$
\begin{aligned}
K_{k \beta}^{\kappa m}(\sigma)= & \frac{(m-1) ! \sigma^{k-m}}{[\sigma \nu(1-u)+\beta]^{k}}\left(\frac{\sigma}{\sigma-2 i p_{1}}\right)^{\kappa} \\
& \times{ }_{2} F_{1}\left(\kappa, k ; m ; \frac{-2 i p_{1} \beta}{\left(\sigma-2 i p_{1}\right)[\sigma \nu(1-u)+\beta]}\right),
\end{aligned}
$$

where ${ }_{2} F_{1}$ for integer $k$ and $m$ reduces to elementary functions, similarly to the one in Eq. (A12). Therefore, taking into account Eq. (A19), the radial matrix elements (A17) may be presented in terms of two-dimensional integrals having a structure similar to that in Eq. (A14) for the radial matrix elements of the FSC amplitude. The final result for $l_{1}=l_{2}+1$ (i.e., $l_{2}=l, l_{1}=l+1$ ) is 


$$
\begin{aligned}
\mathcal{R}_{G}^{(l+1, l)}= & p_{1} \frac{\left|c_{1}\right|}{c_{1}^{*}} \mathcal{S}_{G}^{(l)}+\frac{8^{l+1} Z \nu p_{1}^{l-1} C_{p_{1} l+1} \mathcal{C}_{p_{2} l}(l+1)}{(2 l+1) ! c_{1}^{*}} \\
& \times \int_{0}^{1} d u u^{l-Z \nu} \int_{0}^{1} d x \frac{x^{l+i \eta_{2}}(1-x)^{l-i \eta_{2}}}{\tau^{2 l+3}} \\
& \times\left\{\sum_{k=-1}^{2 l} b_{k}^{(l)}\left[K_{k, \beta_{2}}^{c_{2}, 2 l+2}\left(\lambda^{\prime}\right)-K_{k, \beta_{2}}^{c_{1}, 2 l+2}\left(\lambda^{\prime}\right)\right]\right. \\
& \left.-\frac{1}{\alpha^{2}}\left[K_{2 l, \beta_{1}}^{c_{2}, 2 l+2}(\lambda)-K_{2 l, \beta_{1}}^{c_{1}, 2 l+2}(\lambda)\right]\right\},
\end{aligned}
$$

where $\lambda$ is defined by Eq. (A15) (with $\nu=\nu_{G}$ ) and where

$$
\lambda^{\prime}=i p_{1}+\frac{1}{\nu} \frac{1-\gamma u+\xi \tau(1+u)}{1+\gamma u+\xi \tau(1-u)}
$$

If we define the factor $\sigma \nu(1-u)+\beta \equiv s(\sigma, \beta)$ which appears in Eq. (A24), one can show the nontrivial result that $s\left(\lambda, \beta_{1}\right)=s\left(\lambda^{\prime}, \beta_{2}\right)=i p_{1} \nu(1-u)+1+u$.

For the case $l_{2}=l_{1}+1$ (i.e., $l_{1}=l, l_{2}=l+1$ ), the relation similar to Eq. (A21) is [66]

$$
\begin{aligned}
D(l+1, l) R_{p_{1} l}(r)= & -p_{1} \frac{c_{1}^{*}}{\left|c_{1}\right|} R_{p_{1} l+1}(r)-\frac{2 Z C_{p_{1} l}}{(2 l+3) !}\left(2 p_{1} r\right)^{l} e^{-i p_{1} r} \\
& \times\left[c_{2} \Phi\left(c_{3} ; 2 l+4 ; 2 i p_{1} r\right)\right. \\
& \left.+c_{1}^{*} \Phi\left(c_{2}, 2 l+4 ; 2 i p_{1} r\right)\right] .
\end{aligned}
$$

Thus we obtain

$$
\begin{aligned}
\mathcal{R}_{G}^{(l, l+1)}= & -p_{1} \frac{c_{1}^{*}}{\left|c_{1}\right|} \mathcal{S}_{G}^{(l+1)}+\frac{8^{l+2} Z \nu p_{1}^{l} C_{p_{1} l} \mathcal{C}_{p_{2} l+1}(l+2)}{(2 l+3) !} \\
& \times \int_{0}^{1} d u u^{l+1-Z \nu} \int_{0}^{1} d x \frac{x^{l+1+i \eta_{2}}(1-x)^{l+1-i \eta_{2}}}{\tau^{2 l+5}} \\
& \times\left\{\sum_{k=-1}^{2 l+2} b_{k}^{(l+1)}\left[c_{2} K_{k, \beta_{2}}^{c_{3}, 2 l+4}\left(\lambda^{\prime}\right)+c_{1}^{*} K_{k, \beta_{2}}^{c_{2}, 2 l+4}\left(\lambda^{\prime}\right)\right]\right. \\
& \left.-\frac{1}{\alpha^{2}}\left[c_{2} K_{2 l+2, \beta_{1}}^{c_{3}, 2 l+4}(\lambda)+c_{1}^{*} K_{2 l+2, \beta_{1}}^{c_{2}, 2 l+4}(\lambda)\right]\right\} .
\end{aligned}
$$

The Eqs. (A14), (A25), (A22), and (A28) present our final results for the radial matrix elements $\mathcal{R}_{F, G}^{\left(l_{1}, l_{2}\right)}$, which may be easily calculated numerically for an arbitrary set of individual photoelectron orbital angular momenta $l_{1}$ and $l_{2}$ $=l_{1} \pm 1$. Note that the integrals over $x$ in these equations that involve the functions $J(\lambda), K(\lambda)$, and $J\left(\lambda_{0}\right)$ (all three of which are independent of $x$ ) may be calculated analytically in terms of elementary functions,

$$
\begin{aligned}
\int_{0}^{1} d x & \frac{x^{l+i \eta_{2}}(1-x)^{l-i \eta_{2}}}{\tau^{2 l+3+k}} \\
= & \frac{\mid \Gamma\left(l+1+\left.i \eta_{2}\right|^{2}\right.}{\left(-i p_{2}\right)^{2 l+3+k}(2 l+1) !} \\
& \times \frac{{ }_{2} F_{1}\left(-1-k, l+1+i \eta_{2} ; 2 l+2 ; 2 /\left(i \eta_{s}+1\right)\right)}{\left(i \eta_{s}+1\right)^{l+1+i \eta_{2}}\left(i \eta_{s}-1\right)^{l+2+k-i \eta_{2}}}
\end{aligned}
$$

where $\tau$ is defined below Eq. (A8), $\eta_{s}=Z_{s} / p_{2}$, and $k=0$ or 2 , so that ${ }_{2} F_{1}$ reduces to simple polynomials. The contributions of integrals of the form in Eq. (A29) to $\mathcal{R}_{F, G}^{\left(l_{1}, l_{2}\right)}$ correspond to the account of only the large- $r$ asymptotic behavior of the correlation potential $u_{p l}(r)$ in Eq. (A6), which is defined by the asymptotic behavior of the function $\chi_{p l}(r)$,

$$
\chi_{p l}(r) \approx \frac{(2 l+2) !}{\tau^{2 l+3}} r^{-l-1}, \quad r \rightarrow \infty .
$$

As can be seen from Eqs. (A2) and (A17), in this approximation the dependence of $\mathcal{R}_{F, G}^{\left(l_{1}, l_{2}\right)}$ on the photoelectron momenta factorizes to the product of two independent functions of $p_{1}$ and $p_{2}$ (in contrast to the exact results). The small- $r$ asymptotic behavior of the function $\chi_{p l}(r)$ in Eq. (A8) is given by

$$
\chi_{p l}(r) \approx \frac{r^{l}}{\tau^{2}}, \quad r \rightarrow 0,
$$

so that a similar factorization appears as well in this approximation.

\section{APPENDIX B: EXPRESSIONS FOR THE TRANSITION AMPLITUDE WITH ACCOUNT OF ALL ANGULAR MOMENTA}

Below we present analytic expressions for the functions $A\left(p_{1}, p_{2}, \theta_{12}\right)$ and $B\left(p_{1}, p_{2}, \theta_{12}\right)$ which enter the transition amplitudes $f_{1,2}$ in Eq. (34) that account exactly for all individual photoelectron orbital angular momenta. Each of the functions $A\left(p_{1}, p_{2}, \theta_{12}\right)$ and $B\left(p_{1}, p_{2}, \theta_{12}\right)$ is the sum of FSC and GSC contributions, labeled by indices $F$ and $G$. The functions $A_{F, G}$ and $B_{F, G}$ involve an integral over the variable $x$ [stemming from the integral representation (30) for the CGF] as well as a two-dimensional integration over $q$ and $\theta_{q}$ of the functions $u_{A}\left(q, \theta_{q}\right)$ and $u_{B}\left(q, \theta_{q}\right)$, respectively, which are defined by

$$
\begin{gathered}
u_{A}\left(q, \theta_{q}\right)=\int_{0}^{2 \pi} v_{\mathbf{p}_{2}}\left(q, \theta_{q}, \varphi_{q}\right) \cos \varphi_{q} d \varphi_{q}, \\
u_{B}\left(q, \theta_{q}\right)=\int_{0}^{2 \pi} v_{\mathbf{p}_{2}}\left(q, \theta_{q}, \varphi_{q}\right) d \varphi_{q},
\end{gathered}
$$

where $v_{\mathbf{p}_{2}}\left(q, \theta_{q}, \varphi_{q}\right)$ is defined in Eq. (32). The scalar product $\left(\mathbf{p}_{2} \cdot \mathbf{q}\right)$ may be written as follows (in the coordinate system whose $z$ axis is directed along $\mathbf{p}_{1}$ and whose $x z$ plane is spanned by $\mathbf{p}_{1}$ and $\mathbf{p}_{2}$ ): 


$$
\left(\mathbf{p}_{2} \cdot \mathbf{q}\right)=p_{2} q\left(\sin \theta \sin \theta_{q} \cos \varphi_{q}+\cos \theta \cos \theta_{q}\right)
$$

Although the integration over $\varphi_{q}$ in the integrals (B1) for $u_{A}$ and $u_{B}$ may be easily performed numerically, they may also be evaluated analytically (by using Eq. (3.682) in Ref. [60] and Eq. (15.4.7) in Ref. [67]) in terms of the Legendre functions of the first kind, $P_{\mu}(z)={ }_{2} F_{1}(-\mu, \mu+1 ; 1 ;(1-z) / 2)$ (which reduce to Legendre polynomials for integer $\mu$ )

$$
\begin{aligned}
u_{A}\left(q, \theta_{q}\right)= & 2 \pi\left[q^{2}-\left(p_{2}+i Z_{s}\right)^{2}\right]^{-i \eta_{2}-1}\left(p_{2}+i Z_{s}\right) \\
& \times \frac{1}{b}\left[c J^{(\alpha-1)}-\eta_{2} J^{(\alpha-2)}\right]-\frac{a}{b} u_{B}\left(q, \theta_{q}\right), \\
u_{B}\left(q, \theta_{q}\right)= & 2 \pi\left[q^{2}-\left(p_{2}+i Z_{s}\right)^{2}\right]^{-i \eta_{2}-1}\left(p_{2}+i Z_{s}\right) \\
& \times\left[c J^{(\alpha)}-\eta_{2} J^{(\alpha-1)}\right], \\
J^{(\alpha)}= & {[(a-b)(a-3 b)]^{-\alpha / 2} P_{\alpha}(z), } \\
\alpha=2 & -i \eta_{2}, \quad z=\frac{a-2 b}{\sqrt{(a-b)(a-3 b)}},
\end{aligned}
$$

where the following notations have been used:

$$
\begin{gathered}
a=Z_{s}^{2}+p_{2}^{2}+q^{2}+2 p_{2} q \cos \theta \cos \theta_{q}, \\
b=2 p_{2} q \sin \theta \sin \theta_{q}, \\
c=\left(Z_{s}-Z\right)\left(p_{2}+i Z_{s}\right)+\eta_{2}\left(Z_{s}^{2}+p_{2}^{2}+q^{2}\right) .
\end{gathered}
$$

The functions $A$ and $B$ for the FSC amplitude may thus be presented as follows:

$$
\begin{aligned}
A_{F}= & \frac{Z_{s} C}{\nu^{2}} \int_{0}^{\infty} q d q \int_{0}^{\pi} d \theta_{q} \sin ^{2} \theta_{q} u_{A}\left(q, \theta_{q}\right)\left(\frac{\partial}{\partial \lambda_{1}}\right)_{0} \\
& \times \int_{0}^{1} \frac{d x x^{1-Z \nu}\left(\alpha_{4}-i p_{1}-Z\right)}{(1-x)^{4}\left(\alpha_{1} \alpha_{2} \alpha_{3}\right)^{2} \alpha_{4}^{3}}\left(1-\frac{i p_{1}}{\alpha_{4}}\right)^{-1-i \eta_{1}}, \\
B_{F}= & -i Z_{s} C \int_{0}^{\infty} d q \int_{0}^{\pi} d \theta_{q} \sin \theta_{q} u_{B}\left(q, \theta_{q}\right)\left(\frac{\partial}{\partial \lambda_{1}}\right)_{0} \\
& \times\left(\frac{\partial}{\partial \lambda}\right)_{0}^{1} \frac{d x x^{-Z \nu}}{(1-x)^{2} \beta_{1} \beta_{2} \beta_{3} \beta_{4}}\left(1-\frac{i p_{1}}{\beta_{4}}\right)^{-i \eta_{1}} .
\end{aligned}
$$

In these expressions $C$ is a normalization factor,

$$
C=\frac{\sqrt{2}}{\pi \nu}\left(Z_{s} / 2 \pi\right)^{3} A_{p_{1}}^{(-)^{*}} A_{p_{2}}^{(-)^{*}},
$$

where the coefficient $A_{p}^{(-)^{*}}$ is defined below Eq. (29), and where we have used the following notations:

$$
X=\frac{1}{2 \nu} \frac{1+x}{1-x}, \quad \nu=\frac{1}{\sqrt{-2 E_{F}}}=\frac{1}{\sqrt{2 \epsilon_{1 s}-p_{1}^{2}-p_{2}^{2}}},
$$

$$
q_{x y}=q \sin \theta_{q}, \quad q_{z}=q \cos \theta_{q} .
$$

In addition, the parameters $\alpha_{i}, \beta_{i}$ are defined by

$$
\begin{gathered}
\alpha_{1}=X+\frac{Z_{s}}{2}, \\
\alpha_{2}=X+\frac{Z_{s}}{2}, \\
\alpha_{3}=X-\lambda_{1}-\frac{i p_{1}}{2}+\frac{i q_{z}}{2}-\frac{x}{\nu^{2}(1-x)^{2} \alpha_{2}}, \\
\alpha_{4}=X-\lambda_{1}+\frac{i p_{1}}{2}-\frac{i q_{z}}{2}-\frac{x}{\nu^{2}(1-x)^{2} \alpha_{1}}+\frac{q_{x y}^{2}}{4 \alpha_{3}}, \\
\beta_{1}=\alpha_{1}-\lambda, \\
\beta_{2}=\alpha_{2}+\lambda,
\end{gathered}
$$$$
\beta_{3}=\alpha_{3} \text { with the substitution } \alpha_{2} \rightarrow \beta_{2} \text {, }
$$$$
\beta_{4}=\alpha_{4} \text { with the substitutions } \alpha_{1} \rightarrow \beta_{1}, \alpha_{3} \rightarrow \beta_{3} \text {. }
$$

The parameters $\lambda_{1}$ and $\lambda$ in the factors $\alpha_{i}$ and $\beta_{i}$ in Eqs. (B5) and (B6) should be set equal to zero after evaluation of the derivatives.

Functions $A$ and $B$ for the ground-state correlation amplitude have forms similar to those in Eqs. (B5) and (B6). For this case the parameter $\nu$ is given by $\nu=1 / \sqrt{-2 E_{G}}$ $=1 / \sqrt{4 \epsilon_{1 s}-p_{2}^{2}}$, and $Z_{s}=Z$. The corresponding results are

$$
\begin{aligned}
A_{G}= & -\frac{Z C}{\nu^{2}} \int_{0}^{\infty} q d q \int_{0}^{\pi} d \theta_{q} \sin ^{2} \theta_{q} u_{A}\left(q, \theta_{q}\right) \\
& \times\left(\frac{\partial}{\partial \lambda_{1}}\right)_{0} \int_{0}^{1} \frac{d x x^{1-Z \nu}}{(1-x)^{4}\left(\gamma_{1} \gamma_{2} \gamma_{3} \gamma_{4}\right)^{2}}\left(1-\frac{i p_{1}}{\gamma_{4}}\right)^{1-i \eta_{1}} \\
B_{G}= & -2 p_{1} C \int_{0}^{\infty} d q \int_{0}^{\pi} d \theta_{q} \sin \theta_{q} u_{B}\left(q, \theta_{q}\right)\left(\frac{\partial}{\partial \lambda_{1}}\right)_{0} \\
& \times \int_{0}^{1} d x \frac{x^{-Z \nu}}{(1-x)^{2}}\left\{\frac{1}{2}\left(\frac{\partial}{\partial \lambda}\right)_{0} \frac{1}{\delta_{1} \delta_{2} \delta_{3} \delta_{4}}\left(1+\frac{i p_{1}}{\delta_{4}}\right)^{i \eta_{1}-1}\right. \\
& \left.-\frac{i \eta_{1}}{\epsilon_{1} \epsilon_{2} \epsilon_{3} \epsilon_{4}^{2}}\left(1+\frac{i p_{1}}{\epsilon_{4}}\right)^{i \eta_{1}-1}\right\}
\end{aligned}
$$

where

$$
\delta_{1}=X-\lambda-\frac{i p_{1}}{2}
$$

$$
\delta_{2}=X-\lambda_{1}+\frac{Z}{2}+\frac{i q_{z}}{2}-\frac{x}{\nu^{2}(1-x)^{2} \delta_{1}},
$$




$$
\begin{gathered}
\delta_{3}=X-\lambda_{1}+\frac{Z}{2}-\frac{i q_{z}}{2}+\frac{q_{x y}^{2}}{4 \delta_{2}}, \\
\delta_{4}=X-\lambda-\frac{i p_{1}}{2}-\frac{x}{\nu^{2}(1-x)^{2} \delta_{3}}, \\
\epsilon_{i}=\left(\delta_{i}\right)_{\lambda=0}, \quad i=1,2,3,4
\end{gathered}
$$

$$
\begin{gathered}
\gamma_{1}=\delta_{1}+\lambda, \quad \gamma_{2}=\delta_{2}, \quad \gamma_{3}=\delta_{3}, \\
\gamma_{4}=\delta_{4}+\lambda+i p_{1} .
\end{gathered}
$$

As may by seen from Eq. (B2), for $\theta=0$ and $\pi$ the function $v\left(q, \theta_{q}, \varphi_{q}\right)$ in Eq. (B1) is independent of $\varphi_{q}$, so that $u_{A}\left(q, \theta_{q}\right)=0$. Therefore, for $\theta=0$ and $\theta=\pi$ only the amplitude $B$ contributes to the amplitude $f$ in Eq. (34).
[1] J. S. Briggs and V. Schmidt, J. Phys. B 33, R1 (2000).

[2] G. C. King and L. Avaldi, J. Phys. B 33, R215 (2000).

[3] J. Berakdar and H. Klar, Phys. Rep. 340, 473 (2001).

[4] F. W. Byron and C. J. Joachain, Phys. Rev. 164, 1 (1967).

[5] R. L. Brown, Phys. Rev. A 1, 586 (1970).

[6] M. S. Yurev, Opt. Spektrosk. 38, 9 (1975) [Opt. Spectrosc. 38, 4 (1975)].

[7] M. Y. Amusia, E. G. Drukarev, V. G. Gorshkov, and M. P. Kazachkov, J. Phys. B 8, 1248 (1975).

[8] S. M. Varnavshikh and L. N. Labzovskii Opt. Spektrosk. 47, 45 (1979) [Opt. Spectrosc. 47, 24 (1979)].

[9] S. L. Carter and H. P. Kelly, Phys. Rev. A 24, 170 (1981).

[10] T. Ishihara, K. Hino, and J. H. McGuire, Phys. Rev. A 44, R6980 (1991).

[11] K. I. Hino, T. Ishihara, F. Shimizu, N. Toshima, and J. H. McGuire, Phys. Rev. A 48, 1271 (1993).

[12] C. Pan and H. P. Kelly, J. Phys. B 28, 5001 (1995).

[13] M. Brauner, J. S. Briggs, and H. Klar, J. Phys. B 22, 2265 (1989).

[14] F. Maulbetsch and J. S. Briggs, Phys. Rev. Lett. 68, 2004 (1992); J. Phys. B 26, 1679 (1993); 27, 4095 (1994).

[15] J. Berakdar and H. Klar, Phys. Rev. Lett. 69, 1175 (1992).

[16] J. Berakdar, H. Klar, A. Huetz, and P. Selles, J. Phys. B 26, 1463 (1993).

[17] Z. Teng and R. Shakeshaft, Phys. Rev. A 47, R3487 (1993).

[18] K. I. Hino, Phys. Rev. A 47, 4845 (1993).

[19] M. Pont, R. Shakeshaft, F. Maulbetsch, and J. S. Briggs, Phys. Rev. A 53, 3671 (1996).

[20] V. Mergel, M. Achler, R. Dörner, K. Khayyat, T. Kambara, Y. Awaya, V. Zoran, B. Nyström, L. Spielberg, J. H. McGuire, J. Feagin, J. Berakdar, Y. Azuma, and H. Schmidt-Böcking, Phys. Rev. Lett. 80, 5301 (1998).

[21] S. P. Lucey, J. Rasch, C. T. Whelan, and H. R. J. Walters, J. Phys. B 31, 1237 (1998).

[22] H. Le Rouzo and C. Dal Cappello, Phys. Rev. A 43, 318 (1991).

[23] D. Proulx and R. Shakeshaft, Phys. Rev. A 48, R875 (1993).

[24] M. A. Kornberg and V. D. Rodriguez, Eur. Phys. J. D 5, 221 (1999).

[25] E. G. Drukarev, Phys. Rev. A 52, 3910 (1995).

[26] S. Keller J. Phys. B 33, L513 (2000); S. Keller (private communication).

[27] O. Schwarzkopf and V. Schmidt, J. Phys. B 28, 2847 (1995); 29, 1877 (1996).

[28] R. Dörner, H. Bräuning, J. M. Feagin, V. Mergel, O. Jagutzki, L. Spielberger, T. Vogt, H. Khemliche, M. H. Prior, J. Ullrich, C. L. Cocke, and H. Schmidt-Böcking, Phys. Rev. A 57, 1074 (1998).
[29] H. Bräuning, R. Dörner, C. L. Cocke, M. H. Prior, B. Krässig, A. S. Kheifets, I. Bray, A. Bräuning-Demian, K. Carnes, S. Dreuil, V. Mergel, P. Richard, J. Ullrich, and H. SchmidtBöcking, J. Phys. B 31, 5149 (1998).

[30] A. Huetz and J. Mazeau, Phys. Rev. Lett. 85, 530 (2000).

[31] M. Achler, V. Mergel, L. Spielberg, R. Dörner, Y. Azuma, and H. Schmidt-Böcking, J. Phys. B 34, 965 (2001).

[32] K. W. Meyer and C. H. Greene, Phys. Rev. A 50, R3573 (1994); K. W. Meyer, C. H. Greene, and B. D. Esry, Phys. Rev. Lett. 78, 4902 (1997).

[33] J. Z. Tang and I. Shimamura, Phys. Rev. A 52, R3413 (1995).

[34] A. S. Kheifets and I. Bray, Phys. Rev. A 54, R995 (1996).

[35] A. S. Kheifets and I. Bray, J. Phys. B 31, L447 (1998).

[36] A. Knapp, A. Kheifets, I. Bray, Th. Weber, A. L. Landers, S. Schössler, T. Jahnke, J. Nickles, S. Kammer, O. Jagutzki, L. Ph. H. Schmidt, T. Osipov, J. Rösch, M. H. Prior, H. SchmidtBöcking, C. L. Cocke, and R. Dörnër, Phys. Rev. Lett. 89, 033004 (2002).

[37] P. Selles, L. Malegat, and A. K. Kazansky, Phys. Rev. A 65, 032711 (2002).

[38] J. Colgan, M. S. Pindzola, and F. Robicheaux, J. Phys. B 34, L457 (2001); J. Colgan and M. S. Pindzola, Phys. Rev. A 65, 032729 (2002).

[39] A. Dalgarno and H. R. Sadeghpour, Phys. Rev. A 46, R3591 (1992).

[40] A. Y. Istomin, N. L. Manakov, and A. F. Starace, J. Phys. B 35, L543 (2002).

[41] N. L. Manakov, S. I. Marmo, and A. V. Meremianin, J. Phys. B 29, 2711 (1996).

[42] P. Lablanquie, J. Mazeau, L. Andric, P. Selles, and A. Huetz, Phys. Rev. Lett. 74, 2192 (1995).

[43] L. Malegat, P. Selles, and A. Huetz, J. Phys. B 30, 251 (1997).

[44] M. Ya. Amusia and A. I. Mikhailov, Zh. Eksp. Teor. Fiz. 111, 862 (1997) [JETP 84, 474 (1997)].

[45] J. Colgan and M. S. Pindzola, Phys. Rev. A 65, 032729 (2002).

[46] G. Turri, L. Avaldi, P. Bolognesi, R. Camilloni, M. Coreno, J. Berakdar, A. S. Kheifets, and G. Stefani, Phys. Rev. A 65, 034702 (2002).

[47] M. A. Kornberg and J. E. Miraglia, Phys. Rev. A 49, 5120 (1994).

[48] L. Hostler, J. Math. Phys. 5, 591 (1964).

[49] D. A. Varshalovich, A. N. Moskalev, and V. K. Khersonskii, Quantum Theory of Angular Momentum (World Scientific, Singapore, 1988).

[50] N. L. Manakov and L. P. Rapoport, Opt. Spektrosk. 33, 998 (1972) [Opt. Spectrosc. 33, 547 (1972)].

[51] N. L. Manakov, S. I. Marmo, and A. G. Fainshtein, Zh. Eksp. 
Teor. Fiz. 108, 1569 (1995) [JETP 81, 860 (1995)].

[52] V. L. Yakhontov and M. Ya. Amusia, Phys. Rev. A 55, 1952 (1997).

[53] S. A. Collins, A. Huetz, T. J. Reddish, D. P. Seccombe, and K. Soejima, Phys. Rev. A 64, 062706 (2001).

[54] S. Cvejanovic, J. P. Wightman, T. J. Reddish, F. Maulbetsch, M. A. MacDonald, A. S. Kheifets, and I. Bray, J. Phys. B 33, 265 (2000).

[55] P. Bolognesi, R. Camilloni, M. Coreno, G. Turri, J. Berakdar, A. S. Kheifets, and L. Avaldi, J. Phys. B 34, 3193 (2001).

[56] C. Dawson, S. Cvejanovic, D. Seccombe, T. J. Reddish, F. Maulbetsch, A. Huetz, J. Mazeau, and A. S. Kheifets, J. Phys. B 34, L525 (2001).

[57] S. A. Collins, S. Cvejanovic, C. Dawson, T. J. Reddish, D. P. Seccombe, A. Huetz, L. Malegat, P. Selles, A. K. Kazansky, A. Danjo, K. Soejima, K. Okuno, and A. Yagishita, Phys. Rev. A 65, 052717 (2002).

[58] M. Reed and B. Simon, Methods of Modern Mathematical Physics IV: Analysis of Operators (Academic, New York, 1978), Sec. XII.2.
[59] J. Midtdal, Phys. Rev. 138, A1010 (1965).

[60] A. S. Kheifets and I. Bray, Phys. Rev. A 58, 4501 (1998).

[61] A. I. Mikhailov, I. A. Mikhailov, V. A. Nefiodov, G. Plunien, and G. Soff, Pis'ma Zh. Eksp. Teor. Fiz. 78, 141 (2003) [JETP Lett. 78, 110 (2003)].

[62] J. A. R. Samson, Phys. Rev. Lett. 65, 2861 (1990).

[63] T. Schneider, P. L. Chocian, and J.-M. Rost, Phys. Rev. Lett. 89, 073002 (2002); T. Pattard, T. Schneider, and J.-M. Rost, J. Phys. B 36, L189 (2003); T. Schneider and J.-M. Rost, Phys. Rev. A 67, 062704 (2003)

[64] I. S. Gradshteyn and I. M. Ryzhik, Table of Integrals, Series and Products (Academic, New York, 1980).

[65] A. Erdelyi, W. Magnus, F. Oberhettinger, and F. G. Tricomi, Higher Transcendental Functions (McGraw-Hill, New York, 1953), Vol. 1.

[66] A. A. Krylovetskii, N. L. Manakov, S. I. Marmo, and A. F. Starace, Zh. Eksp. Teor. Fiz. 122, 1168 (2002) [JETP 95, 1006 (2002)].

[67] M. Abramowitz and I. Stegun, Handbook of Mathematical Functions (Dover, New York, 1970). 